\title{
A Systematic Review of Efficacy, Safety, and Tolerability of Duloxetine
}

\author{
Daniela Rodrigues-Amorim ${ }^{1}$, José Manuel Olivares ${ }^{1,2,3}$, Carlos Spuch ${ }^{1}$ and \\ Tania Rivera-Baltanás ${ }^{4 *}$
}

\begin{abstract}
${ }^{1}$ Translational Neuroscience Research Group, Galicia Sur Health Research Institute (IISGS), University of Vigo, Centro de Investigación Biomédica en Red de Salud Mental (CIBERSAM), Vigo, Spain, ${ }^{2}$ Head of Department of Psychiatry, Health Area of Vigo, Servizo Galego de Saúde (SERGAS), Vigo, Spain, ${ }^{3}$ Director Neuroscience Area, Galicia Sur Health Research Institute (IISGS), Centro de Investigación Biomédica en Red de Salud Mental (CIBERSAM), Vigo, Spain, ${ }^{4}$ Translational Neuroscience Research Group, Galicia Sur Health Research Institute (IISGS), Centro de Investigación Biomédica en Red de Salud Mental (CIBERSAM), Vigo, Spain
\end{abstract}

\section{OPEN ACCESS}

Edited by: Mirko Manchia,

University of Cagliari, Italy

Reviewed by:

Kabirullah Lutfy,

Western University of Health Sciences, United States Anna Brancato,

University of Palermo, Italy

*Correspondence:

Tania Rivera-Baltanás tania.rivera@iisgaliciasur.es

Specialty section: This article was submitted to

Psychopharmacology, a section of the journal

Frontiers in Psychiatry

Received: 27 July 2020 Accepted: 24 September 2020 Published: 23 October 2020

Citation:

Rodrigues-Amorim D, Olivares JM, Spuch C and Rivera-Baltanás T (2020)

A Systematic Review of Efficacy,

Safety, and Tolerability of Duloxetine. Front. Psychiatry 11:554899.

doi: 10.3389/fpsyt.2020.554899
Duloxetine is a serotonin-norepinephrine reuptake inhibitor approved for the treatment of patients affected by major depressive disorder (MDD), generalized anxiety disorder (GAD), neuropathic pain (NP), fibromyalgia (FMS), and stress incontinence urinary (SUI). These conditions share parallel pathophysiological pathways, and duloxetine treatment might be an effective and safe alternative. Thus, a systematic review was conducted following the 2009 Preferred Reporting Items (PRISMA) recommendations and Joanna Briggs Institute Critical (JBI) Appraisals guidelines. Eighty-five studies focused on efficacy, safety, and tolerability of duloxetine were included in our systematic review. Studies were subdivided by clinical condition and evaluated individually. Thus, 32 studies of MDD, 11 studies of GAD, 19 studies of NP, 9 studies of FMS, and 14 studies of SUI demonstrated that the measured outcomes indicate the suitability of duloxetine in the treatment of these clinical conditions. This systematic review confirms that the dual mechanism of duloxetine benefits the treatment of comorbid clinical conditions, and supports the efficacy, safety, and tolerability of duloxetine in short- and long-term treatments.

Keywords: duloxetine, clinical conditions, efficacy, safety, tolerability

\section{INTRODUCTION}

Depression and chronic pain are disabling and often concomitant pathologies; both are currently two of the main public health problems $(1,2)$. Major depressive disorder (MDD) is the most prevalent psychiatric disease and has been recognized as a critical target of intervention in the psychiatric field $(3,4)$. However, depression remains underdiagnosed and consequently, undertreated $(4,5)$. Furthermore, generalized anxiety disorder (GAD), obsessive-compulsive disorder (OCD), and post-traumatic stress disorder (PTSD) are common psychiatric comorbidities with MDD, usually lead to worse prognosis and compromise the remission of MDD symptoms (6).

GAD is one of the most prevalent psychiatric disorders, affecting $6 \%$ of the population during their lifetime $(7,8)$. GAD is a chronic condition that severely affects the quality of life, due to its repercussion on working and social functioning (8). Even though anxiety is a widespread symptom, the diagnosis of GAD requires a complex process of screening for a correct diagnosis (9). Moreover, GAD is usually associated with other clinical conditions such as MDD or pain syndromes, affecting drastically prognosis, and treatment efficacy (10).

Chronic pain is a persistent pain condition with a dual dimension, based on the signaling mechanism pathways: nociceptive and neuropathic pain (NP) (11). Specifically, NP has a strong emotional implication, and has been associated with worse quality of life, and clinically, it is related 
with affective disturbances such as depression, anhedonia, working memory dysfunction, sleep disturbances, anxiety, and impaired cognition (12-14). Moreover, chronic pain involves a stress component that might play a bidirectional predictive role. That is, chronic stressful events produce biochemical and pathophysiologic alterations that lead to stress-related mood disorders, that also may increase the risk of chronic pain or exacerbate it $(14,15)$.

On the other hand, fibromyalgia syndrome (FMS) is a chronic widespread pain condition with high heterogeneity clinically and etiologically $(16,17)$. It is estimated that $4-6 \%$ of adults worldwide suffer from FMS, whose incidence is increased in women (18). The most debilitating symptom of FMS is generalized pain. Other symptoms such as fatigue, sleep disturbances, cognitive impairment, or headache are also part of the core symptoms of FMS (19). Concomitantly, MDD symptoms also overlap with the FMS, as well as GAD that is significantly higher in patients with FMS $(20,21)$.

There is a possible connection between anxiety, depression, and stress urinary incontinence (SUI). Evidence suggests that both anxiety and depression are predictor of SUI onset (22). SUI is characterized by an unintentional urinary leakage due to coughing, exertion or sneezing, which increase the intra-abdominal and bladder pressure that overcome urethral resistance (23). Serotonin (5-HT) pathways are involved in this disorder. Thus, 5-HT induces the urethral sphincter closure by inhibition of the micturition reflex (22).

In this perspective, duloxetine is a potential treatment for these dissimilar clinical conditions, but with shared pathophysiological pathways. Duloxetine is a serotoninnorepinephrine reuptake inhibitor (SNRIs) approved as a first-line drug to treat MDD, GAD, diabetic peripheral neuropathy (DPN), FMS and SUI (24-28). As a SNRIs, duloxetine increases both levels of serotonin and norepinephrine which are directly correlated with adverse events, such as tachycardia, hypertension, among others (29). Pharmacokinetic and pharmacodynamic data of duloxetine have been reported for several studies, whose evidence suggests that duloxetine is generally well-tolerated (30-33). Thus, the main goal of this systematic review was to determine the efficacy, tolerability, and safety of duloxetine in the treatment of the clinical conditions for which it is approved.

\section{METHODOLOGY}

\section{Study Design}

A qualitative systematic review of literature was performed, following the 2009 Preferred Reporting Items for Systematic Reviews and Meta-Analysis (PRISMA) guidelines, and the Joanna Briggs Institute (JBI) critical appraisal checklist for the different types of studies reviewed (34). This systematic review aimed to describe and synthetize the evidence and potential benefits of duloxetine.

\section{Protocol Registration}

The protocol was registered in the international database PROSPERO of the National Institute for Health and Research (NIHR) with the code CRD42020153634.

\section{Eligibility Criteria}

To accomplish this comprehensive systematic review the following inclusion criteria were assumed: all studies written in English and focused on human adults (at least 18 years old) with MDD, GAD, NP, FMS, or SUI (clinical conditions for which duloxetine has approval), published until 01/09/2020. Those studies whose primary outcomes were efficacy, tolerability, and/or safety of duloxetine were included. Studies focused on other psychiatric or neurological condition such as Parkinson's disease, Alzheimer's disease, chronic non-neuropathic pain, bipolar, schizoaffective, and schizophrenia disorders were excluded. Moreover, qualitative research reports were also excluded, as well as reports whose analyses were based on pooled integrative data analysis of randomized control trials (RCTs). Eligible designs included RCTs, non-randomized experimental studies, case-control, and cohort studies, which outcomes were quantitatively measured by social, functional, cognitive, quality of life (QoL), or treatment emergent adverse events (TEAEs) instruments.

\section{Data Sources and Search Strategy}

Studies were selected from PubMed, Medline, Web of Science, and PsycINFO electronic databases, introducing the search terms: "duloxetine" AND "major depressive disorder" OR "MDD"; "duloxetine" AND "generalized anxiety disorder" OR "GAD"; "duloxetine" AND "neuropathic pain"; "duloxetine" AND "fibromyalgia"; and "duloxetine" AND "stress urinary incontinence." Two independent researchers (DRA and JMO) conducted the search strategy, applying the filters described in the inclusion criteria to refine the process and obtain concise results.

\section{Study Selection}

Authors independently screened the reports. Firstly, titles and abstracts were reviewed to evaluate their concordance with our requirements. Secondly, the full-text of the potential studies were screened and appreciated and those that met our inclusion criteria were selected. Finally, 85 studies were included in this systematic review. Discrepancies were resolved through discussion among the authors until consensus was reached.

\section{Data Extraction and Synthesis}

To summarize the relevant information of the selected studies, the authors extracted and performed a Table with the following data: first author and year of publication, number of participants, gender, mean age (years), duloxetine dose per day ( $\mathrm{mg}$ ), duration of the treatment (weeks), diagnosis scales or other clinical measuring instruments, relevant statistical results, type of study, and the principal outcomes. The process of synthesis allowed a critical appraisal of the studies and the effect size calculation based on the statistical data reported by studies. 


\section{RESULTS}

\section{Search Results}

The first stage of the searching process comprised a search in the electronic databases using specific search terms, where 2,661 reports were identified. In the second stage, inclusion criteria were applied, duplicate reports were removed, and 727 records by title and abstract were studied. Three hundred and fortytwo studies were analyzed in the third stage, and their fulltext versions were carefully examined. In this stage, they were 85 eligible studies that met the inclusion criteria and the JBI recommendations (Supplementary Tables 1-4). Finally, in the fourth stage, studies on the different clinical conditions for which duloxetine treatment is approved -32 studies on major depressive disorder (MDD), 11 studies on GAD, 19 studies on NP, 9 studies on fibromyalgia, and 14 studies on SUI were selected (Figure 1).

\section{Study Characteristics}

The eligible studies were examined and categorized by clinical condition. Five clinical conditions were considered for the treatment with duloxetine: MDD, GAD, NP, FMS, and SUI. Thus, eighty-five studies were scrutinized and a total of 34,808 participants were enrolled (25,448 female, 9,108 male; and 581 participants that in their study were not differentiated by gender), with an age range of 18-97 years. The studies included 21,406 patients that were treated with duloxetine with a dose ranged from 20 to $120 \mathrm{mg}$ and a treatment duration of $12 \pm 14.39$ weeks. The main reasons of dropout were adverse events (59.5\%), lack of effectiveness $(20.3 \%)$, patient's decision (9.5\%), loss of followup $(5.4 \%)$, non-adherence to treatment $(2.7 \%)$, hospitalizations (1.3\%), and others (1.3\%). Within adverse events, the most common were nausea (18.13\%), dry mouth (9.69\%), constipation (7.42\%), and somnolence (5.94\%) (Figure 2). Cardiovascular disease was an exclusion criterion of $7 \%$ of the studies, and cardiovascular adverse events (hypertension, tachycardia, myocardial ischemia, increased blood pressure, and arrythmia) were evaluated in $68.2 \%$ of the studies, where $49.4 \%$ reported statistical insignificance for these TEAEs $(P<0.05)$, and $11.8 \%$ showed a correlation between elevated heart rate and duloxetine treatment. Regarding the type of studies, $58.7 \%$ of the studies are RCTs, $25.9 \%$ are cohort studies, $11.8 \%$ are quasi-experimental studies (non-randomized) and 3.5\% are case-control studies.

\section{Major Depressive Disorder}

MDD studies comprised 1,836 patients that were treated with $20-120 \mathrm{mg}$ of duloxetine during $8 \pm 17.05$ weeks. This condition was diagnosed based on the Diagnosis and Statistical Manual of Mental Disorders (DSM) from their third to fifth edition. Efficacy of duloxetine was measured in $78.1 \%$ of the studies using the Hamilton Depression Rating Scale (HAMD), the Geriatric Depression Scale (GDS), the Montgomery and Asberg Depression Rating Scale (MADRS) or the Brief Pain Inventory (BPI) when pain, and MDD were concomitant. Safety of treatment with duloxetine was assessed in $25 \%$ of the studies based on TEAEs, and tolerability was evaluated in $31.3 \%$ of studies (35-66). Twenty-six studies were able to demonstrate the superiority of duloxetine over placebo or other antidepressants such as sertraline, fluvoxamine, venlafaxine, paroxetine, escitalopram, fluoxetine, and bupropion. Five studies did not find statistical significance $(P \geq 0.05)$ regarding the correlation between duloxetine and the outcomes and one study did not obtain significant results when comparing duloxetine with sertraline. Safety and tolerability were evaluated by TEAEs and the most common adverse events (AEs) were nausea, somnolence, dry mouth, hyperhidrosis, constipation, and sedation; patient's dropout rate was $\sim 10 \%$ (Figure 2 ). This result was not significant in most of studies, concluding that duloxetine was safe and well-tolerated (see Table 1).

\section{Generalized Anxiety Disorder}

Eleven studies focused on GAD were included in this systematic review, which involved 2,608 patients treated with $20-120 \mathrm{mg}$ of duloxetine with an average duration of treatment of 10 \pm 6.59 weeks. All studies used the DSM to accomplish the diagnosis. Clinical evidence was based on the correlation between the Hamilton Anxiety Rating Scale (HAMA) and the outcomes. Therefore, all studies found statistical significance ( $P \leq 0.05$ ) when measured the efficacy ( $90.9 \%$ of the studies), safety and tolerability (both $27.3 \%$ of the studies) (67-77). TEAEs were measured and nausea, dry mouth, dizziness, and somnolence were reported as the most frequent AEs (Figure 2). One study reported suicidal ideation, although no statistical significance was found between duloxetine and placebo groups (69). Duloxetine was more effective, safe and tolerated than placebo or other antidepressants (escitalopram and venlafaxine) (Table 1).

\section{Neuropathic Pain}

The selected NP studies reported the use of duloxetine in the treatment of peripheral neuropathy induced by chemotherapy, diabetic peripheral neuropathic pain (DPNP), radiculopathy and neuropathic pain associated with multiple sclerosis (NPMS) (Table 1). NP condition was diagnosed using specific criteria of pain detection, being the BPI the most commonly applied instrument. TEAEs were the measure for safety and tolerability. The dose of duloxetine applied was ranged from 20 to $120 \mathrm{mg}$ during $12 \pm 14.53$ weeks. The core of the studies focused on 4,627 patients with NP, where the efficacy, safety, and tolerability of duloxetine were compared with placebo, anticonvulsant treatments (pregabalin and gabapentin), other antidepressants (venlafaxine and amitriptyline), or even with different daily doses of duloxetine. Thus, $78.9 \%$ of the studies reported efficacy outcomes, 47.4 and $21.1 \%$ of the studies described safety and tolerability of duloxetine, respectively (78-96). Three studies did not show statistical significance regarding efficacy, safety, and tolerability of duloxetine against anticonvulsants $(P \geq 0.05)$. Nausea, somnolence, insomnia, constipation, and decreased appetite were the most prevalent TEAEs (Figure 2). A minority of patients discontinued the treatment with duloxetine due to AEs (12.2\%). Nevertheless, $84.2 \%$ of the studies supported the evidence of duloxetine as first-line treatment of NP conditions. 


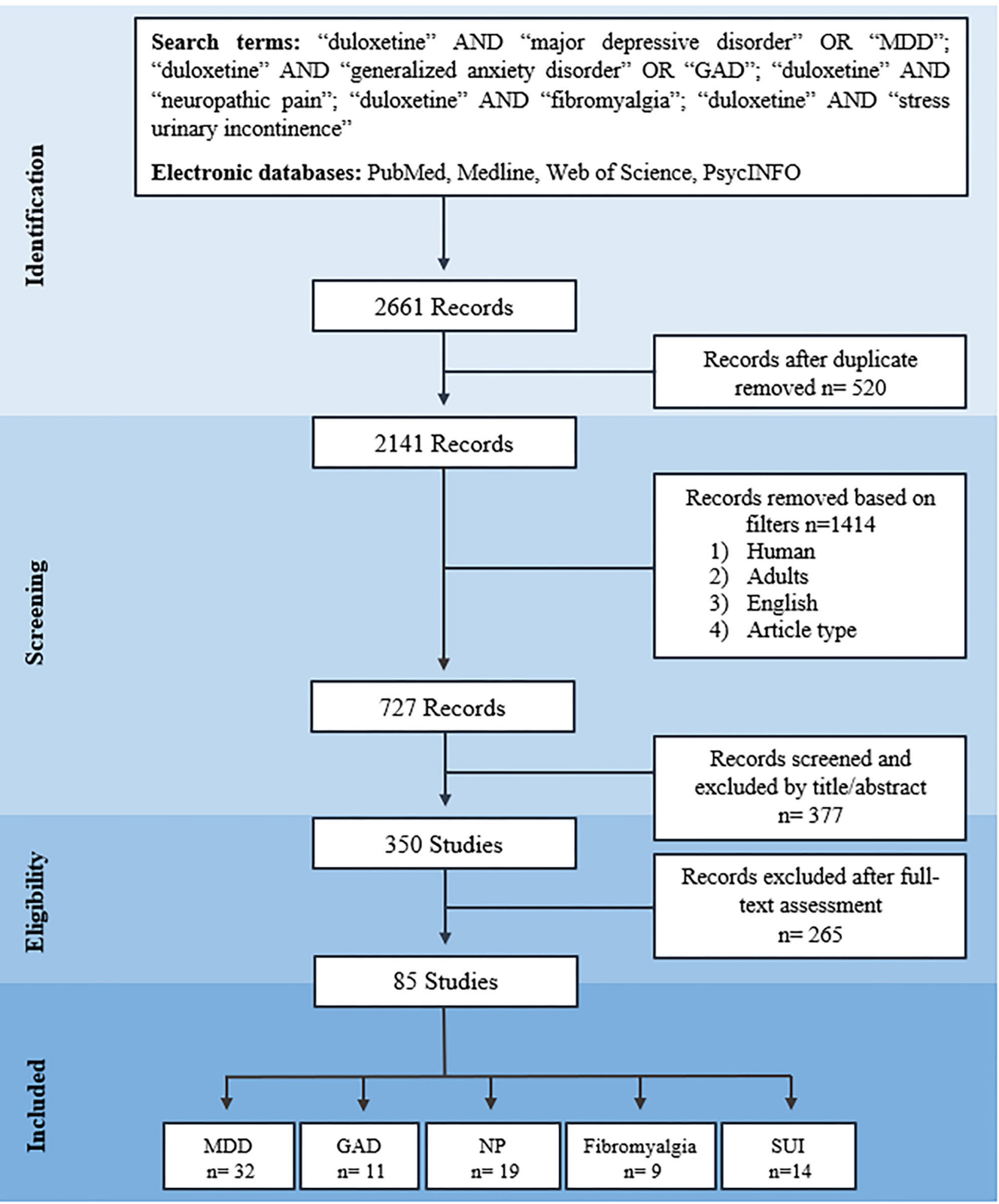

FIGURE 1 | PRISMA 2009 flow diagram of search process. MDD, major depressive disorder; GAD, generalized anxiety disorder; NP, neuropathic pain; SUI, stress urinary incontinence. 


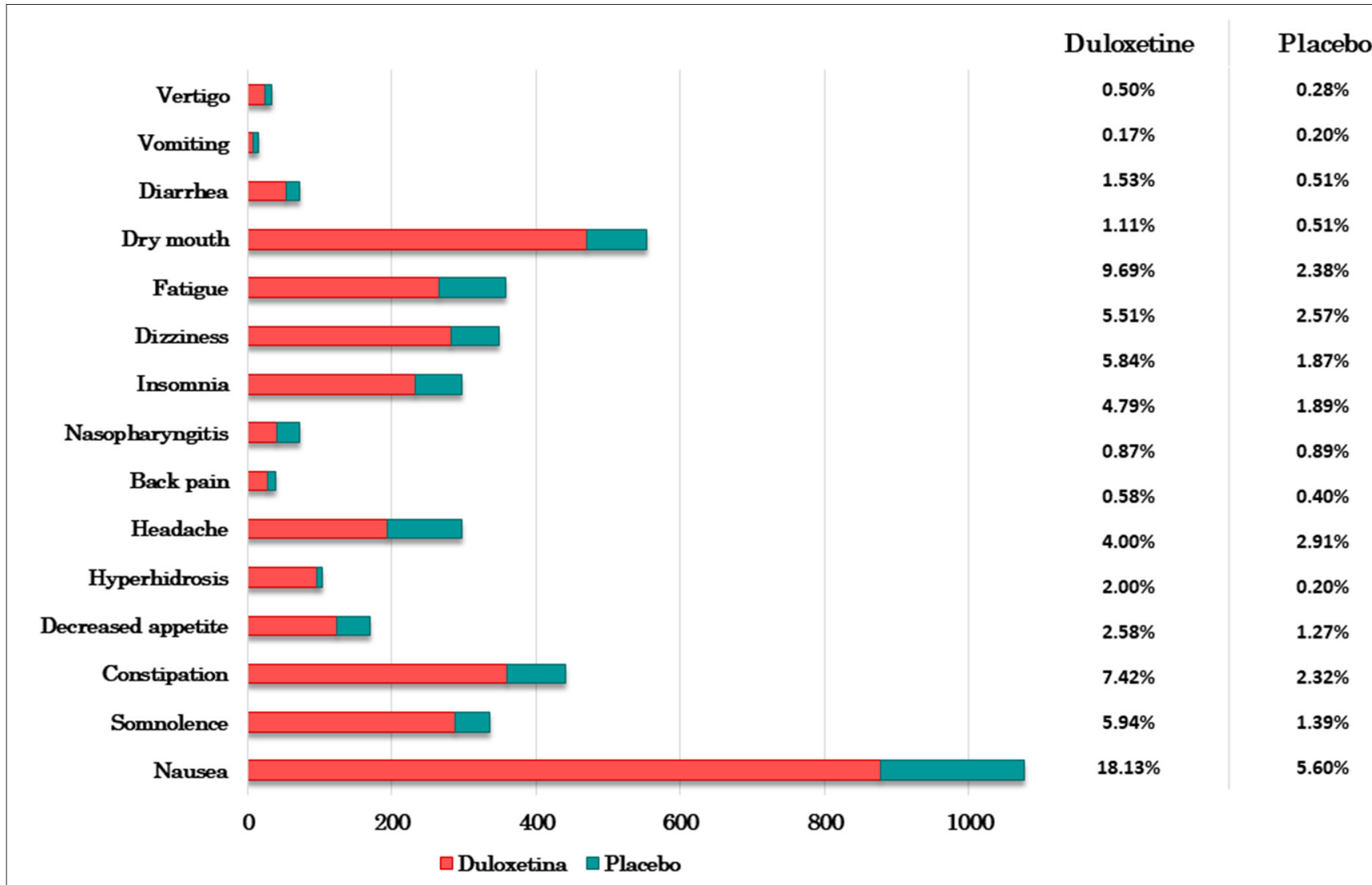

FIGURE 2 | Adverse events of duloxetine-treated patients and placebo patients. Bar graph of the most common adverse effects vs. the number (N) of participants who developed them ( $N_{\text {total }}$ duloxetine-treated patients $=4,848$ and $N_{\text {total }}$ placebo patients $\left.=3,536\right)$. The table shows the corresponding percentage.

\section{Fibromyalgia}

Nine studies focusing on FMS were assessed and eligible. They involved a total of 1,918 patients. The average duration of treatment was $24 \pm 13.78$ weeks and the dose of duloxetine oscillated from 20 to $120 \mathrm{mg}$ per day (Table 1). The BPI scale and Fibromyalgia Impact Questionnaire (FIQ) were the instruments employed to analyse the outcomes. Most studies $(77.8 \%)$ evaluated the efficacy of duloxetine, and $55.6 \%$ provided data of the treatment safety $(97-104,119)$. Statistically significant results were obtained in seven studies, where duloxetine improved symptomatology, reducing the pain impact registered by BPI. In contrast, two studies reported no statistical differences relative to BPI change average and cognitive improvement in fibromyalgia patients. The duloxetine treatment was related to $\sim 17 \%$ of adverse effects. Taking all these studies into account, duloxetine showed to be safe and effective in FMS treatment.

\section{Stress Urinary Incontinence}

Fourteen studies that involved 6,395 patients (99.5\% female and $0.5 \%$ male) were assessed. Duloxetine doses were between 20 and $120 \mathrm{mg}$ per day. Treatment duration was $12 \pm 6.72$ weeks and the Incontinence Episode Frequency (IEF) and Incontinence Quality of Life (I-QoL) were the instruments to measure the outcomes. All studies evaluated efficacy of duloxetine; 71.4 and
$7.1 \%$ of the studies measured safety and tolerability, respectively (105-118). Treatment was discontinued in $22.1 \%$ of patients regarding TEAEs, being the most common fatigue, nausea, constipation, and dry mouth (Figure 2). These AEs tend to improve and disappear with continuing duloxetine therapy. In conclusion, significant results were found in all studies, proving the efficacy, safety, and tolerability of duloxetine in the treatment of SUI.

\section{Quality Assessment}

A systematic review summarizes the evidence of the relevant literature, however, an unbiased search of studies without an explicit assess strategy could lead to a poor scientific report. The relevance and quality of the studies selected and included in this systematic review fulfilled the PRISMA recommendations (120), and JBI critical appraisal guidelines. The JBI is an evidence-based organization that develops strategies to conduct and perform a high quality systematic reviews (121). Thus, the quality determination of the studies included indicates that our research minimizes the risk of selection bias. Furthermore, a good systematic review relies on the studies it contains. Therefore, the inclusion of RCTs and clinical trials reduce the probability of bias due to their strict methodology (122). 
TABLE 1 | Characteristics of the selected studies and included in the systematic review.

\begin{tabular}{|c|c|c|c|c|c|c|c|c|c|c|}
\hline References & $\mathbf{N}^{\circ}$ participants & Gender & $\begin{array}{l}\text { Years (mean + } \\
\text { SD) }\end{array}$ & $\begin{array}{l}\text { Dose } \\
\text { duloxetine }\end{array}$ & $\begin{array}{l}\text { Treatment } \\
\text { duration }\end{array}$ & $\begin{array}{l}\text { Diagnosis scales, } \\
\text { measures }\end{array}$ & $P$-value & G Hedges & Type of study & Outcome \\
\hline \multicolumn{11}{|c|}{ Major depressive disorder } \\
\hline $\begin{array}{l}\text { De Donatis et al. } \\
\text { (66) }\end{array}$ & $\begin{array}{l}\mathrm{n}^{\circ} \text { duloxetine }=66 \\
\text { Total } \mathrm{n}^{\circ}=66\end{array}$ & $40 \mathrm{~F} / 26 \mathrm{M}$ & $56.42 \pm 14.55$ & $60 \mathrm{mg}$ & 12 weeks & $\begin{array}{l}\text { DSM-IV, HAMD21, } \\
\text { serum concentration }\end{array}$ & $P<0.001$ & 1.907 & Cohort study & $\begin{array}{l}\text { Treatment response } \\
\text { MDD } \\
\text { Serum concentration } \\
\text { duloxetine }\end{array}$ \\
\hline Mowla et al. (65) & $\begin{array}{l}\mathrm{n}^{\circ} \text { duloxetine }=26 \\
\mathrm{n}^{\circ} \text { sertraline }=28 \\
\text { Total } \mathrm{n}^{\circ}=54\end{array}$ & $32 \mathrm{~F} / 22 \mathrm{M}$ & 42.3 & $40-60 \mathrm{mg}$ & 6 weeks & $\begin{array}{l}\text { DSM-V, SCID-I, } \\
\text { HAMD21, CGI-2 }\end{array}$ & $P=0.463$ & 0.391 & RCT double-blind & $\begin{array}{l}\text { Compare the effects } \\
\text { of sertraline with } \\
\text { duloxetine in MDD } \\
\text { HAMD21 }\end{array}$ \\
\hline Buoli et al. (64) & $\begin{array}{l}\mathrm{n}^{\circ} \text { escitalopram }=10 \\
\mathrm{n}^{\circ} \text { citalopram }=19 \\
\mathrm{n} \text {. paroxetine }=23 \\
\mathrm{n}^{\circ} \text { mirtazapine }=8 \\
\mathrm{n}^{\circ} \text { fluoxetine }=13 \\
\mathrm{n}^{\circ} \text { clomipramine }=8 \\
\mathrm{n}^{\circ} \text { sertraline }=14 \\
\mathrm{n}^{\circ} \text { trazodone }=6 \\
\mathrm{n}^{\circ} \text { duloxetine }=10 \\
\mathrm{n}^{\circ} \text { venlafaxine }=12 \\
\mathrm{n}^{\circ} \text { fluvoxamine }=12 \\
\mathrm{n}^{\circ} \text { amitriptyline }=10 \\
\mathrm{n}^{\circ} \text { bupropion }=5 \\
\text { Total } \mathrm{n}^{\circ}=150\end{array}$ & $115 \mathrm{~F} / 35 \mathrm{M}$ & $51.03 \pm 13.83$ & $\begin{array}{c}65.50 \pm 15.89 \\
(\mathrm{mg})\end{array}$ & 96 weeks & DSM-V, SCID-I & $P<0.01$ & $\begin{array}{l}2.984 \text { (fluvoxamine } \\
3.623 \text { (bupropion) }\end{array}$ & eClinical trial & $\begin{array}{l}\text { Efficacy at long-term } \\
\text { treatment of MDD } \\
\text { Breslow's test }\end{array}$ \\
\hline $\begin{array}{l}\text { Robinson et al. } \\
\text { (63) }\end{array}$ & $\begin{array}{l}\mathrm{n}^{\circ} \text { duloxetine }=204 \\
\mathrm{n}^{\circ} \text { placebo }=95 \\
\text { Total } \mathrm{n}^{\circ}=299\end{array}$ & $191 \mathrm{~F} / 108 \mathrm{M}$ & $73.01 \pm 6.26$ & $60-120 \mathrm{mg}$ & 24 weeks & $\begin{array}{l}\text { DSM-IV, HAMD17, } \\
\text { GDS, CGI-S, PGI-I, } \\
\text { BPI, NRS, TEAES }\end{array}$ & $P=0.004$ & 4.545 & RCT double-blind & $\begin{array}{l}\text { Efficacy in elderly } \\
\text { patients with MDD } \\
\text { GDS }\end{array}$ \\
\hline $\begin{array}{l}\text { Martinez et al. } \\
\text { (60) }\end{array}$ & $\begin{array}{l}\mathrm{n}^{\circ} \text { duloxetine }=372 \\
\mathrm{n}^{\circ} \text { SSRIs }=378 \\
\text { Total } \mathrm{n}^{\circ}=750\end{array}$ & $536 \mathrm{~F} / 214 \mathrm{M}$ & $44.3 \pm 13.0$ & $30-60 \mathrm{mg}$ & 12 weeks & $\begin{array}{l}\text { DSM-IV, QIDS-SR, } \\
\text { HAMD17, BPI, SDS }\end{array}$ & $P<0.01$ & 4.250 & RCT non-blinded & $\begin{array}{l}\text { Efficacy in } \\
\text { moderate-to-severe } \\
\text { depressive episode } \\
\text { HAMD17 total }\end{array}$ \\
\hline Oakes et al. (61) & $\begin{array}{l}\mathrm{n}^{\circ} \text { duloxetine }=261 \\
\mathrm{n}^{\circ} \text { placebo }=131 \\
\text { Total } \mathrm{n}^{\circ}=392\end{array}$ & $256 \mathrm{~F} / 136 \mathrm{M}$ & $44.7 \pm 12.2$ & $60 \mathrm{mg}$ & 8 weeks & $\begin{array}{l}\text { DSM-IV, HAMD17, } \\
\text { SDS, SASS, CGI-S }\end{array}$ & $P<0.001$ & 6.577 & $\begin{array}{l}\text { RCT double-blind } \\
\text { phase IV }\end{array}$ & $\begin{array}{l}\text { Efficacy } \\
\text { HAMD17 }\end{array}$ \\
\hline Rosso et al. (62) & $\begin{array}{l}\mathrm{n}^{\circ} \text { duloxetine }=25 \\
\mathrm{n}^{\circ} \text { bupropion }=21 \\
\text { Total } \mathrm{n}^{\circ}=46\end{array}$ & $31 \mathrm{~F} / 15 \mathrm{M}$ & $47.6 \pm 12.6$ & $120 \mathrm{mg}$ & 6 weeks & $\begin{array}{l}\text { DSM-IV, HAMD17, } \\
\text { CGI-I, GAF }\end{array}$ & $P=0.793$ & 0.076 & RCT double-blind & $\begin{array}{l}\text { Efficacy } \\
\text { HAMD17 }\end{array}$ \\
\hline Brecht et al. (48) & $\begin{array}{l}\mathrm{n}^{\circ} \text { duloxetine } 60 \\
=167 \mathrm{n}^{\circ} \text { duloxetine } \\
120=171 \text { Total } \mathrm{n}^{\circ} \\
=338\end{array}$ & $251 \mathrm{~F} / 87 \mathrm{M}$ & $44.8 \pm 13.3$ & $60-120 \mathrm{mg}$ & 8 weeks & $\begin{array}{l}\text { DSM-IV, MADRS, } \\
\text { HDRS-6, CGI-S, } \\
\text { TEAEs }\end{array}$ & $\begin{array}{c}P=0.88 \\
\text { TEAEs }>10 \%\end{array}$ & 0.019 & RCT double-blind & $\begin{array}{l}\text { Efficacy and safety } \\
\text { MADRS }\end{array}$ \\
\hline
\end{tabular}


TABLE 1 | Continued

\begin{tabular}{|c|c|c|c|c|c|c|c|c|c|c|}
\hline References & $\mathbf{N}^{\circ}$ participants & Gender & $\begin{array}{l}\text { Years (mean + } \\
\text { SD) }\end{array}$ & $\begin{array}{c}\text { Dose } \\
\text { duloxetine }\end{array}$ & $\begin{array}{l}\text { Treatment } \\
\text { duration }\end{array}$ & $\begin{array}{l}\text { Diagnosis scales, } \\
\text { measures }\end{array}$ & $P$-value & G Hedges & Type of study & Outcome \\
\hline Gaynor et al. (59) & $\begin{array}{l}\mathrm{n}^{\circ} \text { duloxetine }=262 \\
\mathrm{n}^{\circ} \text { placebo }=266 \\
\text { Total } \mathrm{n}^{\circ}=528\end{array}$ & $302 \mathrm{~F} / 226 \mathrm{M}$ & $46.2 \pm 13$ & $60 \mathrm{mg}$ & 8 weeks & $\begin{array}{l}\text { DSM-IV, MINI, } \\
\text { MADRS, BPI, SDS, } \\
\text { CGI-S, PGI, TEAES }\end{array}$ & $\begin{array}{c}P<0.001 \\
\text { TEAEs }=5 \%\end{array}$ & 6.167 & RCT double-blind & $\begin{array}{l}\text { Efficacy and tolerability } \\
\text { MADRS }\end{array}$ \\
\hline $\begin{array}{l}\text { Sagman et al. } \\
\text { (58) }\end{array}$ & $\begin{array}{l}\mathrm{n}^{\circ} \text { duloxetine } \\
\text { responders }=115 \mathrm{n}^{\circ} \\
\text { duloxetine } \\
\text { non-responders }=91 \\
\text { Total } \mathrm{n}^{\circ}=242\end{array}$ & $182 \mathrm{~F} / 60 \mathrm{M}$ & $44.9 \pm 12.5$ & 60-120 mg & 8 weeks & $\begin{array}{l}\text { DSM-IV, BPI-SF, } \\
\text { HAMD17 }\end{array}$ & $P=0.042$ & - & $\begin{array}{l}\text { Clinical trial } \\
\text { open-label }\end{array}$ & $\begin{array}{l}\text { Switching to } \\
\text { duloxetine treatment } \\
\text { BPI-SF }\end{array}$ \\
\hline $\begin{array}{l}\text { Herrera-Guzmán } \\
\text { et al. (56) }\end{array}$ & $\begin{array}{l}\mathrm{n}^{\circ} \text { duloxetine }=37 \\
\mathrm{n}^{\circ} \text { escitalopram }=36 \\
\mathrm{n}^{\circ} \text { control }=37 \\
\text { Total } \mathrm{n}^{\circ}=110\end{array}$ & $78 \mathrm{~F} / 32 \mathrm{M}$ & $33.21 \pm 8.61$ & $60 \mathrm{mg}$ & 24 weeks & $\begin{array}{l}\text { DSM-IV, MINI, } \\
\text { HAMD17, WAIS III, } \\
\text { SWM, RVIP, MTS, } \\
\text { Stroop test, ID/ED, } \\
\text { SOC }\end{array}$ & $P<0.001$ & 4.864 & Case-control study & $\begin{array}{l}\text { Efficacy in improving } \\
\text { attention and } \\
\text { executive functions } \\
\text { HAMD17 }\end{array}$ \\
\hline $\begin{array}{l}\text { Volonteri et al. } \\
\text { (57) }\end{array}$ & $\begin{array}{l}\mathrm{n}^{\circ} \text { duloxetine }=45 \\
\text { Total } \mathrm{n}^{\circ}=45\end{array}$ & $29 \mathrm{~F} / 16 \mathrm{M}$ & $59.6 \pm 12.79$ & 30-120 mg & 12 weeks & $\begin{array}{l}\text { DSM-IV, HRSD, } \\
\text { CGI-S, BDI, VAS, } \\
\text { AEs }\end{array}$ & $\begin{array}{l}P<0.001 \\
A E s=9 \%\end{array}$ & 9.402 & $\begin{array}{l}\text { Naturalistic } \\
\text { open-label study }\end{array}$ & $\begin{array}{l}\text { Clinical response and } \\
\text { tolerability } \\
\text { HRSD }\end{array}$ \\
\hline $\begin{array}{l}\text { Perahia et al. } \\
\text { (44) }\end{array}$ & $\begin{array}{l}\mathrm{n}^{\circ} \text { duloxetine }=146 \\
\mathrm{n}^{\circ} \text { placebo }=142 \\
\text { Total } \mathrm{n}^{\circ}=288\end{array}$ & $206 \mathrm{~F} / 82 \mathrm{M}$ & $47.1 \pm 12.8$ & 60-120 mg & $\begin{array}{l}52 \text { weeks } \\
\text { (maintenance } \\
\text { phase) }\end{array}$ & $\begin{array}{l}\text { DSM-IV, MINI, } \\
\text { HAMD17, CGI-S, } \\
\text { PGI-I, SDS, VAS, } \\
\text { SF-36, SQ-SS, } \\
\text { TEAES }\end{array}$ & $\begin{array}{c}P<0.001 \\
P_{\text {TEAEs }}>0.05\end{array}$ & 5.380 & RCT double-blind & $\begin{array}{l}\text { Recurrence of MDD } \\
\text { Safety and tolerability } \\
\text { HAMD17 }\end{array}$ \\
\hline Karp et al. (53) & $\begin{array}{l}n^{\circ} \text { duloxetine }=40 \\
\text { Total } n^{\circ}=40\end{array}$ & $26 \mathrm{~F} / 14 \mathrm{M}$ & $74.4 \pm 7.0$ & $120 \mathrm{mg}$ & 16 weeks & $\begin{array}{l}\text { DSM-IV, SCID, } \\
\text { MMSE, HAMD17, } \\
\text { UKU, AES }\end{array}$ & $\begin{aligned} P & <0.01 \\
\mathrm{AEs} & =12.5 \%\end{aligned}$ & 0.029 & $\begin{array}{l}\text { Open label } \\
\text { Cohort study }\end{array}$ & $\begin{array}{l}\text { Tolerability } \\
\text { UKU }\end{array}$ \\
\hline $\begin{array}{l}\text { Kornstein et al. } \\
(54)\end{array}$ & $\begin{array}{l}\mathrm{n}^{\circ} \text { duloxetine } \\
\text { non-remitters } 60= \\
130 \\
\mathrm{n}^{\circ} \text { duloxetine non } \\
\text { remitters } 120=118 \\
\mathrm{n}^{\circ} \text { duloxetine } \\
\text { remitters } 60=193 \\
\text { Total } \mathrm{n}^{\circ}=441\end{array}$ & $275 \mathrm{~F} / 166 \mathrm{M}$ & $44.7 \pm 12.77$ & 30-120 mg & 16 weeks & $\begin{array}{l}\text { DSM-IV, HAMD17, } \\
\text { IDS-C-30, } \\
\text { QIDS-C-16, BPI-SF, } \\
\text { VAS, CGI-S, PGI, } \\
\text { TEAES }\end{array}$ & $P \leq 0.05$ & $\begin{array}{l}8.885 \\
8.491\end{array}$ & RCT double-blind & $\begin{array}{l}\text { Efficacy } \\
\text { HAMD17 }\end{array}$ \\
\hline $\begin{array}{l}\text { Perahia et al. } \\
\text { (43) }\end{array}$ & $\begin{array}{l}\mathrm{n}^{\circ} \text { duloxetine direct } \\
\text { switch }=183 \\
\mathrm{n}^{\circ} \text { duloxetine } \\
\text { start-taper switch }= \\
185 \\
\text { Total } \mathrm{n}^{\circ}=368\end{array}$ & $283 \mathrm{~F} / 85 \mathrm{M}$ & $49.05 \pm 12.8$ & 60-120 mg & 8 weeks & $\begin{array}{l}\text { DSM-IV, HAMD17, } \\
\text { CGI-S, EQ-5D, VAS, } \\
\text { SQ-SS, SF-36, } \\
\text { TEAEs }\end{array}$ & $\begin{array}{l}P \leq 0.001 \\
P \leq 0.001\end{array}$ & - & $\begin{array}{l}\text { RCT open-label } \\
\text { non-inferiority study }\end{array}$ & $\begin{array}{l}\text { Efficacy and tolerability } \\
\text { HAMD17 }\end{array}$ \\
\hline $\begin{array}{l}\text { Perahia et al. } \\
\text { (42) }\end{array}$ & $\begin{array}{l}\mathrm{n}^{\circ} \text { duloxetine }=330 \\
\mathrm{n}^{\circ} \text { venlafaxine }=337 \\
\text { Total } \mathrm{n}^{\circ}=667\end{array}$ & $450 \mathrm{~F} / 217 \mathrm{M}$ & $44.3 \pm 12.8$ & 60-120 mg & 12 weeks & $\begin{array}{l}\text { DSM-IV, MINI, } \\
\text { HAMD17, HAMA, } \\
\text { CGI-S, PGI-I, TEAES }\end{array}$ & $P=0.440$ & 1.084 & RCT double-blind & $\begin{array}{l}\text { Global benefit-risk } \\
\text { HAMD17 }\end{array}$ \\
\hline
\end{tabular}


TABLE 1 | Continued

\begin{tabular}{|c|c|c|c|c|c|c|c|c|c|c|}
\hline References & $\mathbf{N}^{\circ}$ participants & Gender & $\begin{array}{l}\text { Years (mean + } \\
\text { SD) }\end{array}$ & $\begin{array}{c}\text { Dose } \\
\text { duloxetine }\end{array}$ & $\begin{array}{l}\text { Treatment } \\
\text { duration }\end{array}$ & $\begin{array}{l}\text { Diagnosis scales, } \\
\text { measures }\end{array}$ & $P$-value & G Hedges & Type of study & Outcome \\
\hline Raskin et al. (51) & $\begin{array}{l}\mathrm{n}^{\circ} \text { duloxetine }=207 \\
\mathrm{n}^{\circ} \text { placebo }=104 \\
\text { Total } \mathrm{n}^{\circ}=311\end{array}$ & $185 \mathrm{~F} / 146 \mathrm{M}$ & $72.6 \pm 5.7$ & $60 \mathrm{mg}$ & 8 weeks & DSM-IV, HAMD17 & $\begin{array}{l}P<0.001 \\
P=0.014 \\
P=0.042\end{array}$ & $\begin{array}{c}2.355 \\
\text { (dry mouth) } \\
2.091 \text { (Nausea) } \\
2.219 \text { (Diarrhea) }\end{array}$ & RCT double-blind & $\begin{array}{l}\text { Safety and tolerability } \\
\text { Adverse effects }\end{array}$ \\
\hline Volpe (55) & $\begin{array}{l}\mathrm{n}^{\circ} \text { duloxetine }=30 \\
\text { Total } \mathrm{n}^{\circ}=30\end{array}$ & $28 \mathrm{~F} / 2 \mathrm{M}$ & $41 \pm 8$ & $60 \mathrm{mg}$ & 8 weeks & $\begin{array}{l}\text { DSM-IV, MADRS, } \\
\text { VAS, } \\
\text { WHOQoL-BREF, } \\
\text { AEs }\end{array}$ & $\begin{array}{c}P<0.001 \\
\mathrm{AEs}=6.7 \%\end{array}$ & 2.874 & $\begin{array}{l}\text { Open label } \\
\text { Cohort study }\end{array}$ & $\begin{array}{l}\text { Efficacy and tolerability } \\
\text { MADRS }\end{array}$ \\
\hline Brecht et al. (46) & $\begin{array}{l}\mathrm{n}^{\circ} \text { duloxetine }=162 \\
\mathrm{n}^{\circ} \text { placebo }=165 \\
\text { Total } \mathrm{n}^{\circ}=327\end{array}$ & $241 \mathrm{~F} / 86 \mathrm{M}$ & 48.1 & $60 \mathrm{mg}$ & 8 weeks & $\begin{array}{l}\text { DSM-IV, MINI, } \\
\text { MADRS, BPI-SF, } \\
\text { CGI-S, TEAEs }\end{array}$ & $\begin{array}{c}P=0.0008 \\
\text { TEAEs }>10 \%\end{array}$ & - & RCT double-blind & $\begin{array}{l}\text { Efficacy and safety } \\
\text { BPI-SF }\end{array}$ \\
\hline Lee et al. (47) & $\begin{array}{l}\mathrm{n}^{\circ} \text { duloxetine }=238 \\
\mathrm{n}^{\circ} \text { paroxetine }=240 \\
\text { Total } \mathrm{n}^{\circ}=478\end{array}$ & $333 \mathrm{~F} / 145 \mathrm{M}$ & $39.0 \pm 13.95$ & $60 \mathrm{mg}$ & 8 weeks & $\begin{array}{l}\text { DSM-IV, HAMD17, } \\
\text { VAS, CGI-S, PGI-I, } \\
\text { TEAEs }\end{array}$ & $\begin{array}{c}P=0.218 \\
P_{\text {TEAES }}>0.05\end{array}$ & - & RCT double-blind & $\begin{array}{l}\text { Efficacy and safety } \\
\text { HAMD17 }\end{array}$ \\
\hline Pigott et al. (49) & $\begin{array}{l}\mathrm{n}^{\circ} \text { duloxetine }=273 \\
\mathrm{n}^{\circ} \text { escitalopram }= \\
274 \\
\mathrm{n}^{\circ} \text { placebo }=137 \\
\text { Total } \mathrm{n}^{\circ}=684\end{array}$ & $446 \mathrm{~F} / 238 \mathrm{M}$ & $41.1 \pm 11.6$ & $60-120 \mathrm{mg}$ & 8 months & $\begin{array}{l}\text { DSM-IV, MINI, } \\
\text { MADRS, CGI-S, } \\
\text { PGI-I, HAMA, } \\
\text { CSFQ, AEs }\end{array}$ & $\begin{array}{c}P=0.44 \\
\mathrm{AEs}=12.8 \%\end{array}$ & $\begin{array}{l}0.774 \\
3.272\end{array}$ & RCT double-blind & $\begin{array}{l}\text { Efficacy, safety, and } \\
\text { tolerability } \\
\text { HAMD17 }\end{array}$ \\
\hline Raskin et al. (50) & $\begin{array}{l}\mathrm{n}^{\circ} \text { duloxetine }=207 \\
\mathrm{n}^{\circ} \text { placebo }=104 \\
\text { Total } \mathrm{n}^{\circ}=311\end{array}$ & $185 \mathrm{~F} / 126 \mathrm{M}$ & $72.6 \pm 5.7$ & $60 \mathrm{mg}$ & 8 weeks & $\begin{array}{l}\text { DSM-IV, HAMD17, } \\
\text { MMSE, CGI-S, } \\
\text { WAIS-III, VAS, CCS, } \\
\text { GDS }\end{array}$ & $P<0.02$ & - & RCT double-blind & $\begin{array}{l}\text { Efficacy on cognition, } \\
\text { depression, and pain } \\
\text { cCS }\end{array}$ \\
\hline Wise et al. (52) & $\begin{array}{l}\mathrm{n}^{\circ} \text { duloxetine }=828 \\
\mathrm{n}^{\circ} \text { placebo }=416 \\
\text { Total } \mathrm{n}^{\circ}=1,244\end{array}$ & $740 \mathrm{~F} / 504 \mathrm{M}$ & $72.8 \pm 5.6$ & $60 \mathrm{mg}$ & 8 weeks & $\begin{array}{l}\text { DSM-IV, MMSE, } \\
\text { CCS, GDS, } \\
\text { HAMD17, CGI-S, } \\
\text { VAS, SF-36, TEAES }\end{array}$ & $\begin{array}{c}P=0.013 \\
\text { TEAEs }=9.7 \%\end{array}$ & - & RCT double-blind & $\begin{array}{l}\text { Safety and tolerability } \\
\text { with comorbidities } \\
\text { CCS }\end{array}$ \\
\hline Fava et al. (45) & $\begin{array}{l}\mathrm{n}^{\circ} \text { duloxetine } 60 \mathrm{QD} \\
=58 \mathrm{n}^{\circ} \text { duloxetine } \\
60 \mathrm{BID}=29 \text { Total } \mathrm{n}^{\circ} \\
=87\end{array}$ & $69 \mathrm{~F} / 18 \mathrm{M}$ & $43.8 \pm 11.17$ & $60-120 \mathrm{mg}$ & 12 weeks & $\begin{array}{l}\text { DSM-IV, HAMD17, } \\
\text { CGI-S, VAS }\end{array}$ & $P<0.001$ & 0.465 & RCT double-blind & $\begin{array}{l}\text { Depression relapses } \\
\text { HAMD17 }\end{array}$ \\
\hline $\begin{array}{l}\text { Perahia et al. } \\
\text { (41) }\end{array}$ & $\begin{array}{l}\mathrm{n}^{\circ} \text { duloxetine }=136 \\
\mathrm{n}^{\circ} \text { placebo }=142 \\
\text { Total } \mathrm{n}^{\circ}=278\end{array}$ & $202 \mathrm{~F} / 76 \mathrm{M}$ & $45.7 \pm 12.69$ & $60 \mathrm{mg}$ & 26 weeks & $\begin{array}{l}\text { DSM-IV, MINI, } \\
\text { HRSD17, CGI-S }\end{array}$ & $P \leq 0.05$ & 0.675 & RCT double-blind & $\begin{array}{l}\text { Relapse prevention } \\
\text { Relapses }\end{array}$ \\
\hline $\begin{array}{l}\text { Perahia et al. } \\
(40)\end{array}$ & $\begin{array}{l}\mathrm{n}^{\circ} \text { duloxetine } 40 \mathrm{BID} \\
=93 \\
\mathrm{n}^{\circ} \text { duloxetine } 60 \mathrm{BID} \\
=103 \\
\mathrm{n}^{\circ} \text { placebo }=99 \\
\mathrm{n}^{\circ} \text { paroxetine }=97 \\
\text { Total } \mathrm{n}^{\circ}=392\end{array}$ & $273 \mathrm{~F} / 119 \mathrm{M}$ & $45.43 \pm 11.37$ & $80-120 \mathrm{mg}$ & 8 weeks & $\begin{array}{l}\text { DSM-IV, MINI, } \\
\text { HAMD17, CGI-S, } \\
\text { MADRS, HAMA, } \\
\text { VAS }\end{array}$ & $P \leq 0.05$ & $\begin{array}{l}2.600 \\
3.200 \\
2.200\end{array}$ & RCT double-blind & $\begin{array}{l}\text { Efficacy } \\
\text { HAMD17 }\end{array}$ \\
\hline
\end{tabular}


TABLE 1 | Continued

\begin{tabular}{|c|c|c|c|c|c|c|c|c|c|c|}
\hline References & $N^{\circ}$ participants & Gender & $\begin{array}{c}\text { Years (mean + } \\
\text { SD) }\end{array}$ & $\begin{array}{c}\text { Dose } \\
\text { duloxetine }\end{array}$ & $\begin{array}{l}\text { Treatment } \\
\text { duration }\end{array}$ & $\begin{array}{l}\text { Diagnosis scales, } \\
\text { measures }\end{array}$ & $P$-value & G Hedges & Type of study & Outcome \\
\hline Burt et al. (39) & $\begin{array}{l}\mathrm{n}^{\circ} \text { duloxetine }=55 \\
\mathrm{n}^{\circ} \text { placebo }=59 \\
\text { Total } \mathrm{n}^{\circ}=114\end{array}$ & $114 \mathrm{~F}$ & 47.7 & $60 \mathrm{mg}$ & 9 weeks & $\begin{array}{l}\text { DSM-IV, HAMD17, } \\
\text { CGI-S, PGI-I, VAS, } \\
\text { SSI, QLDS }\end{array}$ & $P<0.001$ & 0.686 & RCT double-blind & $\begin{array}{l}\text { Efficacy in women } \\
\text { HAMD17 }\end{array}$ \\
\hline $\begin{array}{l}\text { Goldstein et al. } \\
\text { (36) }\end{array}$ & $\begin{array}{l}\mathrm{n}^{\circ} \text { duloxetine } 20 \mathrm{BID} \\
=86 \\
\mathrm{n}^{\circ} \text { duloxetine } 40 \mathrm{BID} \\
=91 \\
\mathrm{n}^{\circ} \text { placebo }=89 \\
\mathrm{n}^{\circ} \text { paroxetine }=87 \\
\text { Total } \mathrm{n}^{\circ}=353\end{array}$ & $217 \mathrm{~F} / 136 \mathrm{M}$ & $41 \pm 11$ & $40-80 \mathrm{mg}$ & 8 weeks & $\begin{array}{l}\text { DSM-IV, HAMD17, } \\
\text { VAS, CGI-I, PGI-I, } \\
\text { QLDS }\end{array}$ & $\begin{array}{l}P=0.002 \\
P=0.034 \\
P=0.285\end{array}$ & - & RCT double-blind & $\begin{array}{l}\text { Improvement of } \\
\text { emotional and painful } \\
\text { physical symptoms } \\
\text { HAMD17 }\end{array}$ \\
\hline Detke et al. (37) & $\begin{array}{l}\mathrm{n}^{\circ} \text { duloxetine }=123 \\
\mathrm{n}^{\circ} \text { placebo }=122 \\
\text { Total } \mathrm{n}^{\circ}=245\end{array}$ & $163 \mathrm{~F} / 82 \mathrm{M}$ & $42.44 \pm 13.74$ & $60 \mathrm{mg}$ & 9 weeks & $\begin{array}{l}\text { DSM-IV, MINI, } \\
\text { HAMD17, CGI-S, } \\
\text { PGI-I, QLDS, AES }\end{array}$ & $\begin{array}{c}P<0.001 \\
A E s=13.8 \%\end{array}$ & - & RCT double-blind & $\begin{array}{l}\text { Efficacy and tolerability } \\
\text { HAMD17 }\end{array}$ \\
\hline $\begin{array}{l}\text { Goldstein et al. } \\
\text { (38) }\end{array}$ & $\begin{array}{l}\mathrm{n}^{\circ} \text { duloxetine }=70 \\
\mathrm{n}^{\circ} \text { fluoxetine }=33 \\
\mathrm{n}^{\circ} \text { placebo }=70 \\
\text { Total } \mathrm{n}^{\circ}=173\end{array}$ & $111 \mathrm{~F} / 63 \mathrm{M}$ & $42.3 \pm 10.8$ & $40-120 \mathrm{mg}$ & 8 weeks & $\begin{array}{l}\text { DSM-IV, MINI, } \\
\text { HAMD17, CGI-S, } \\
\text { MADRS, PGI, } \\
\text { HAMA, AES }\end{array}$ & $\begin{array}{c}P=0.009 \\
\mathrm{AEs}=4.3 \%\end{array}$ & - & RCT double-blind & $\begin{array}{l}\text { Efficacy, safety, and } \\
\text { tolerability } \\
\text { HAMD17 }\end{array}$ \\
\hline Berk et al. (35) & $\begin{array}{l}\mathrm{n}^{\circ} \text { duloxetine }=93 \\
\text { Total } \mathrm{n}^{\circ}=93\end{array}$ & $62 \mathrm{~F} / 31 \mathrm{M}$ & 38 & $20 \mathrm{mg}$ & 6 weeks & $\begin{array}{l}\text { DSM-III, HAMD17, } \\
\text { CGI-I, PGI, }\end{array}$ & $\begin{array}{c}-16.4 \pm 6.7 \\
\text { (change) }\end{array}$ & 2.565 & $\begin{array}{l}\text { Open label } \\
\text { uncontrolled trial }\end{array}$ & $\begin{array}{l}\text { Efficacy } \\
\text { HAMD17 }\end{array}$ \\
\hline \multicolumn{11}{|c|}{ Generalized depressive disorder } \\
\hline Alaka et al. (67) & $\begin{array}{l}\mathrm{n}^{\circ} \text { duloxetine }=151 \\
\mathrm{n}^{\circ} \text { placebo }=140 \\
\text { Total } \mathrm{n}^{\circ}=291\end{array}$ & $226 \mathrm{~F} / 65 \mathrm{M}$ & $71.4 \pm 5.4$ & $30-120 \mathrm{mg}$ & 10 weeks & $\begin{array}{l}\text { DSM-IV, HAMA, } \\
\text { SDS, HADS, CGIII, } \\
\text { TEAEs }\end{array}$ & $\begin{array}{l}P<0.001 \\
\text { TEAEs }=9 \%\end{array}$ & 6.461 & RCT double-blind & $\begin{array}{l}\text { Efficacy and safety } \\
\text { HAMA }\end{array}$ \\
\hline Bodkin et al. (68) & $\begin{array}{l}\mathrm{n}^{\circ} \text { duloxetine }=216 \\
\mathrm{n}^{\circ} \text { placebo }=213 \\
\text { Total } \mathrm{n}^{\circ}=429\end{array}$ & $257 \mathrm{~F} / 172 \mathrm{M}$ & $45.0 \pm 13.2$ & $60-120 \mathrm{mg}$ & 26 weeks & $\begin{array}{l}\text { DSM -IV, HAMA, } \\
\text { CGI-I, MINI, HADS, } \\
\text { SDS, SQ-SS, VAS }\end{array}$ & $\begin{array}{l}P=0.028 \\
P<0.001\end{array}$ & $\begin{array}{l}1.097 \\
1.650\end{array}$ & RCT double-blind & $\begin{array}{l}\text { Relapses } \\
\text { HAMA-1, VAS }\end{array}$ \\
\hline Pierò et al. (69) & $\begin{array}{l}\mathrm{n}^{\circ} \text { duloxetine }=23 \\
\mathrm{n}^{\circ} \text { escitalopram }=20 \\
\text { Total } \mathrm{n}^{\circ}=43\end{array}$ & $31 \mathrm{~F} / 12 \mathrm{M}$ & $35.3 \pm 17.4$ & $60 \mathrm{mg}$ & 26 weeks & $\begin{array}{l}\text { DSM-IV, HAMA, } \\
\text { HDRS, CGI, GAF }\end{array}$ & $P<0.001$ & 0.374 & $\begin{array}{l}\text { Clinical trial } \\
\text { non-randomized }\end{array}$ & $\begin{array}{l}\text { Effectiveness of } \\
6 \text {-months treatment } \\
\text { with escitalopram and } \\
\text { duloxetine } \\
\text { HAMA }\end{array}$ \\
\hline Wu et al. (70) & $\begin{array}{l}\mathrm{n}^{\circ} \text { duloxetine }=108 \\
\mathrm{n}^{\circ} \text { placebo }=102 \\
\text { Total } \mathrm{n}^{\circ}=210\end{array}$ & $106 \mathrm{~F} / 104 \mathrm{M}$ & $37.3 \pm 11.9$ & $60-120 \mathrm{mg}$ & 15 weeks & $\begin{array}{l}\text { DSM-IV, CAS, RDS, } \\
\text { CGI-S, SDS, } \\
\text { HADS-A, HAMA, } \\
\text { TEAES }\end{array}$ & $\begin{array}{c}P=0.006 \\
P_{\text {TEAEs }}<0.05\end{array}$ & 0.237 & $\begin{array}{l}\text { RCT double-blind } \\
\text { phase III }\end{array}$ & $\begin{array}{l}\text { Efficacy, tolerability, } \\
\text { and safety } \\
\text { HADS-A }\end{array}$ \\
\hline Nicolini et al. (71) & $\begin{array}{l}\mathrm{n}^{\circ} \text { duloxetine } 20= \\
158 \\
\mathrm{n}^{\circ} \text { duloxetine } 60-120 \\
=158 \\
\mathrm{n}^{\circ} \text { venlafaxine }=169 \\
\mathrm{n}^{\circ} \text { placebo }=170 \\
\text { Total } \mathrm{n}^{\circ}=581\end{array}$ & - & 42.8 & $20-120 \mathrm{mg}$ & 10 weeks & $\begin{array}{l}\text { DSM-IV, HAMA, } \\
\text { HADS, CAS, CGI-I }\end{array}$ & $P \leq 0.001$ & 5.286 & RCT double-blind & $\begin{array}{l}\text { Symptoms } \\
\text { improvement } \\
\text { HAMA }\end{array}$ \\
\hline
\end{tabular}


TABLE 1 | Continued

\begin{tabular}{|c|c|c|c|c|c|c|c|c|c|c|}
\hline References & $\mathbf{N}^{\circ}$ participants & Gender & $\begin{array}{l}\text { Years (mean + } \\
\text { SD) }\end{array}$ & $\begin{array}{l}\text { Dose } \\
\text { duloxetine }\end{array}$ & $\begin{array}{l}\text { Treatment } \\
\text { duration }\end{array}$ & $\begin{array}{l}\text { Diagnosis scales, } \\
\text { measures }\end{array}$ & $P$-value & G Hedges & Type of study & Outcome \\
\hline $\begin{array}{l}\text { Allgulander et al. } \\
\text { (72) }\end{array}$ & $\begin{array}{l}\mathrm{n}^{\circ} \text { duloxetine }=320 \\
\mathrm{n}^{\circ} \text { venlafaxine }=333 \\
\mathrm{n}^{\circ} \text { placebo }=331 \\
\text { Total } \mathrm{n}^{\circ}=984\end{array}$ & $596 \mathrm{~F} / 388 \mathrm{M}$ & $41.6 \pm 13.2$ & $60-120 \mathrm{mg}$ & 10 weeks & $\begin{array}{l}\text { DSM-IV, MINI, } \\
\text { HADS, CAS, RDS, } \\
\text { CGI-S }\end{array}$ & $P \leq 0.001$ & - & RCT double-blind & $\begin{array}{l}\text { Duloxetine vs. } \\
\text { Venlafaxine efficacy } \\
\text { HAMA }\end{array}$ \\
\hline $\begin{array}{l}\text { Davidson et la. } \\
\text { (73) }\end{array}$ & $\begin{array}{l}\mathrm{n}^{\circ} \text { duloxetine }=42 \\
\mathrm{n}^{\circ} \text { placebo }=28 \\
\text { Total } \mathrm{n}^{\circ}=70\end{array}$ & $38 \mathrm{~F} / 42 \mathrm{M}$ & $70.1 \pm 4.3$ & $60-120 \mathrm{mg}$ & 9-10 weeks & $\begin{array}{l}\text { DSM-IV, MINI, } \\
\text { HAMA, HADS, CAS, } \\
\text { RDS, CGI-S, TEAES }\end{array}$ & $\begin{array}{c}P=0.029 \\
P_{\text {TEAES }}<0.05\end{array}$ & 3.164 & RCT double-blind & $\begin{array}{l}\text { Efficacy and tolerability } \\
\text { HAMA }\end{array}$ \\
\hline Pollack et al. (74) & $\begin{array}{l}\mathrm{n}^{\circ} \text { duloxetine }=668 \\
\mathrm{n}^{\circ} \text { placebo }=495 \\
\text { Total } \mathrm{n}^{\circ}=1,163\end{array}$ & $753 \mathrm{~F} / 410 \mathrm{M}$ & $42.5 \pm 13.3$ & $60-120 \mathrm{mg}$ & 4 weeks & $\begin{array}{l}\text { DSM-IV, HAMA, } \\
\text { CGI-S, SDS }\end{array}$ & $P<0.001$ & - & RCT double-blind & $\begin{array}{l}\text { Early improvement } \\
\text { HAMA }\end{array}$ \\
\hline Russell et al. (75) & $\begin{array}{l}\mathrm{n}^{\circ} \text { duloxetine }=208 \\
\mathrm{n}^{\circ} \text { placebo }=146 \\
\text { Total } \mathrm{n}^{\circ}=354\end{array}$ & $247 \mathrm{~F} / 107 \mathrm{M}$ & $42.1 \pm 12.7$ & $60-120 \mathrm{mg}$ & 12 weeks & $\begin{array}{l}\text { DSM-IV, MINI, } \\
\text { HAMA, VAS, HDAS, } \\
\text { CAS, RDS, CGI-S }\end{array}$ & $P=0.017$ & - & $\begin{array}{l}\text { RCT double-blind } \\
\text { phase III }\end{array}$ & $\begin{array}{l}\text { Efficacy } \\
\text { HAMA }\end{array}$ \\
\hline Rynn et al. (76) & $\begin{array}{l}\mathrm{n}^{\circ} \text { duloxetine }=168 \\
\mathrm{n}^{\circ} \text { placebo }=159 \\
\text { Total } \mathrm{n}^{\circ}=327\end{array}$ & $202 F / 125 M$ & $42.2 \pm 13.9$ & $60-120 \mathrm{mg}$ & 10 weeks & $\begin{array}{l}\text { DSM-IV, HAMA, } \\
\text { CGI-S, HADS, CAS, } \\
\text { RDS, TEAES }\end{array}$ & $\begin{array}{c}P=0.023 \\
P_{\text {TEAEs }}<0.05\end{array}$ & - & RCT double-blind & $\begin{array}{l}\text { Efficacy and safety } \\
\text { HAMA }\end{array}$ \\
\hline $\begin{array}{l}\text { Hartford et al. } \\
\text { (77) }\end{array}$ & $\begin{array}{l}\mathrm{n}^{\circ} \text { duloxetine }=162 \\
\mathrm{n}^{\circ} \text { venlafaxine }=164 \\
\mathrm{n}^{\circ} \text { placebo }=161 \\
\text { Total } \mathrm{n}^{\circ}=487\end{array}$ & $305 \mathrm{~F} / 182 \mathrm{M}$ & $40.4 \pm 13.6$ & $60-120 \mathrm{mg}$ & 10 weeks & $\begin{array}{l}\text { DSM-IV, SIGH-A, } \\
\text { HADS, CAS, CGI-S, } \\
\text { HAMA, TEAES }\end{array}$ & $\begin{array}{c}P \leq 0.01 \\
\text { TEAEs }=5 \%\end{array}$ & $\begin{array}{l}3.838 \\
4.791\end{array}$ & $\begin{array}{l}\text { RCT double-blind } \\
\text { phase II }\end{array}$ & $\begin{array}{l}\text { Efficacy and tolerability } \\
\text { HAMA }\end{array}$ \\
\hline & Total $n^{\circ}=487$ & & & & & & & & & \\
\hline \multicolumn{11}{|l|}{ Neuropathic pain } \\
\hline $\begin{array}{l}\text { Salehifar et al. } \\
\text { (78) }\end{array}$ & $\begin{array}{l}\mathrm{n}^{\circ} \text { duloxetine }=42 \\
\mathrm{n}^{\circ} \text { pregabalin }=40 \\
\text { Total } \mathrm{n}^{\circ}=82\end{array}$ & $82 \mathrm{~F}$ & $48.7 \pm 9.63$ & $30-60 \mathrm{mg}$ & 6 weeks & $\begin{array}{l}\text { VAS, NCl-CTCAE } \\
\text { v4.03, PNQ, AES }\end{array}$ & $P<0.001$ & $\begin{array}{l}1.647 \\
1.676 \\
1.587\end{array}$ & $\begin{array}{l}\text { RCT double-blind } \\
\text { phase II }\end{array}$ & $\begin{array}{l}\text { Efficacy and safety of } \\
\text { pregabalin and } \\
\text { duloxetine in } \\
\text { taxane-induced } \\
\text { peripheral neuropathy } \\
\text { VAS, NCI-CTCAE } \\
\text { V4.03, PNQ }\end{array}$ \\
\hline Jha et al. (79) & $\begin{array}{l}\mathrm{n}^{\circ} \text { duloxetine }=9 \\
\mathrm{n}^{\circ} \text { pregabalin }=25 \\
\text { Total } \mathrm{n}^{\circ}=34\end{array}$ & $18 \mathrm{~F} / 16 \mathrm{M}$ & $55.8 \pm 8.59$ & 20-30 mg & 16 weeks & $\begin{array}{l}\text { VAS, SF-MPQ, } \\
\text { Mc-Gill, NRS, DN-4, } \\
\text { AEs }\end{array}$ & $P<0.001$ & - & Cohort study & $\begin{array}{l}\text { Efficacy, safety, and } \\
\text { tolerability of } \\
\text { pregabalin compared } \\
\text { to duloxetine in DPNP } \\
\text { Mc-Gill }\end{array}$ \\
\hline $\begin{array}{l}\text { Farshchian et al. } \\
\text { (80) }\end{array}$ & $\begin{array}{l}\mathrm{n}^{\circ} \text { duloxetine }=52 \\
\mathrm{n}^{\circ} \text { venlafaxine }=52 \\
\mathrm{n}^{\circ} \text { placebo }=52 \\
\text { Total } \mathrm{n}^{\circ}=156\end{array}$ & $124 \mathrm{~F} / 32 \mathrm{M}$ & $57.4 \pm 14.5$ & $30 \mathrm{mg}$ & 4 weeks & RTOG criteria & $P<0.05$ & - & RCT double-blind & $\begin{array}{l}\text { Effects of venlafaxine } \\
\text { vs. duloxetine on } \\
\text { chemotherapy- } \\
\text { induced peripheral } \\
\text { neuropathy }\end{array}$ \\
\hline $\begin{array}{l}\text { Schukro et al. } \\
\text { (81) }\end{array}$ & $\begin{array}{l}\mathrm{n}^{\circ} \text { duloxetine }=14 \\
\mathrm{n}^{\circ} \text { placebo }=11 \\
\text { Total } \mathrm{n}^{\circ}=25\end{array}$ & $21 \mathrm{~F} / 20 \mathrm{M}$ & $57.9 \pm 13.4$ & $120 \mathrm{mg}$ & 4 weeks & $\begin{array}{l}\text { VAS, pain DETECT } \\
\text { questionnaire, BDI, } \\
\text { SF-36 }\end{array}$ & $P=0.001$ & 0.675 & RCT double-blind & $\begin{array}{l}\text { Efficacy of duloxetine } \\
\text { in low back pain with } \\
\text { radicular pain } \\
\text { VAS }\end{array}$ \\
\hline
\end{tabular}


TABLE 1 | Continued

\begin{tabular}{|c|c|c|c|c|c|c|c|c|c|c|}
\hline References & $\mathbf{N}^{\circ}$ participants & Gender & $\begin{array}{l}\text { Years (mean + } \\
\text { SD) }\end{array}$ & $\begin{array}{c}\text { Dose } \\
\text { duloxetine }\end{array}$ & $\begin{array}{l}\text { Treatment } \\
\text { duration }\end{array}$ & $\begin{array}{l}\text { Diagnosis scales, } \\
\text { measures }\end{array}$ & $P$-value & G Hedges & Type of study & Outcome \\
\hline Yasuda et al. (82) & $\begin{array}{l}n^{\circ} \text { duloxetine } 40= \\
129 \\
n^{\circ} \text { duloxetine } 60= \\
129 \\
\text { Total } n^{\circ}=258\end{array}$ & $62 \mathrm{~F} / 196 \mathrm{M}$ & $60.1 \pm 10.0$ & $40-60 \mathrm{mg}$ & 52 weeks & BPI, PGI-I, AEs & $\begin{array}{l}P<0.0001 \\
A E \leq 13.6 \%\end{array}$ & $\begin{array}{l}3.273 \\
3.473\end{array}$ & RCT double-blind & $\begin{array}{l}\text { Long-term efficacy } \\
\text { and safety: duloxetine } \\
\text { in diabetic neuropathic } \\
\text { pain } \\
\text { BPI }\end{array}$ \\
\hline Gao et al. (83) & $\begin{array}{l}\mathrm{n}^{\circ} \text { duloxetine }=203 \\
\mathrm{n}^{\circ} \text { placebo }=202 \\
\text { Total } \mathrm{n}^{\circ}=405\end{array}$ & $223 F / 182 M$ & $61.6 \pm 9.7$ & $60 \mathrm{mg}$ & 12 weeks & $\begin{array}{l}\text { BPI-S, PGI-I, SDS, } \\
\text { QIDS-SR, TEAES }\end{array}$ & $\begin{array}{c}P=0.030 \\
\text { TEAEs }=8.4 \%\end{array}$ & 3.071 & $\begin{array}{l}\text { RCT double-blind } \\
\text { phase III }\end{array}$ & $\begin{array}{l}\text { Efficacy and safety: } \\
\text { duloxetine in diabetic } \\
\text { neuropathic pain } \\
\text { BPI-S }\end{array}$ \\
\hline $\begin{array}{l}\text { Happich et al. } \\
\text { (84) }\end{array}$ & $\begin{array}{l}\mathrm{n}^{\circ} \text { duloxetine }=931 \\
\mathrm{n}^{\circ} \text { pregabalin }=248 \\
\mathrm{n}^{\circ} \text { gabapentin }=351 \\
\text { Total } \mathrm{n}^{\circ}=1,530\end{array}$ & $794 \mathrm{~F} / 736 \mathrm{M}$ & $64.0 \pm 11.66$ & $60 \mathrm{mg}$ & 36 weeks & $\begin{array}{l}\text { BPI, CGI, PGI, } \\
\text { HADS, SF-12, SDS }\end{array}$ & $\begin{array}{l}P=0.029 \\
P<0.001\end{array}$ & $\begin{array}{l}0.175 \\
0.531\end{array}$ & Cohort study & $\begin{array}{l}\text { The effectiveness of } \\
\text { duloxetine in DPNP } \\
\text { BPI }\end{array}$ \\
\hline Irving et al. (85) & $\begin{array}{l}\mathrm{n}^{\circ} \text { duloxetine }=138 \\
\mathrm{n}^{\circ} \text { pregabalin }=134 \\
\mathrm{n}^{\circ} \text { duloxetine }+ \\
\text { gabapentin }=135 \\
\text { Total } \mathrm{n}^{\circ}=407\end{array}$ & $165 \mathrm{~F} / 242 \mathrm{M}$ & $60.9 \pm 10.2$ & $60 \mathrm{mg}$ & 12 weeks & $\begin{array}{l}\text { TEAEs, LSEQ, } \\
\text { CSFQ, TEAEs }\end{array}$ & $\begin{array}{c}P>0.05 \\
P_{\text {TEAES }}=0.04\end{array}$ & $\begin{array}{l}0.172 \\
0.187\end{array}$ & $\begin{array}{l}\text { RCT open-label } \\
\text { non-inferiority study }\end{array}$ & $\begin{array}{l}\text { Safety and tolerability } \\
\text { in DPNP } \\
\text { TEAEs }\end{array}$ \\
\hline Vollmer et al. (86) & $\begin{array}{l}\mathrm{n}^{\circ} \text { duloxetine }=118 \\
\mathrm{n}^{\circ} \text { placebo }=121 \\
\text { Total } \mathrm{n}^{\circ}=239\end{array}$ & $179 \mathrm{~F} / 60 \mathrm{M}$ & $50.8 \pm 9.7$ & $30-60 \mathrm{mg}$ & 6 weeks & $\begin{array}{l}\text { DSM-IV, MINI, } \\
\text { C-SSRS, BDI-II, } \\
\text { CGI-S, BPI, } \\
\text { MS-QoL-54, PGI-I, } \\
\text { MFIS, TEAES }\end{array}$ & $\begin{array}{c}P=0.001 \\
\text { TEAEs }=13.6 \%\end{array}$ & 4.606 & RCT double-blind & $\begin{array}{l}\text { Efficacy and tolerability } \\
\text { neuropathic pain in } \\
\text { multiple sclerosis } \\
\text { API }\end{array}$ \\
\hline Smith et al. (87) & $\begin{array}{l}\mathrm{n}^{\circ} \text { duloxetine }=109 \\
\mathrm{n}^{\circ} \text { placebo }=111 \\
\text { Total } \mathrm{n}^{\circ}=220\end{array}$ & $138 \mathrm{~F} / 82 \mathrm{M}$ & $60 \pm 10.4$ & $60 \mathrm{mg}$ & 12 weeks & BPI-SF & $P=0.003$ & 0.513 & $\begin{array}{l}\text { RCT double-blind } \\
\text { phase III }\end{array}$ & $\begin{array}{l}\text { Effects of duloxetine } \\
\text { on chemotherapy- } \\
\text { induced peripheral } \\
\text { neuropathy } \\
\text { BPI-SF }\end{array}$ \\
\hline $\begin{array}{l}\text { Tesfaye et al. } \\
\text { (88) }\end{array}$ & $\begin{array}{l}\mathrm{n}^{\circ} \text { duloxetine }=401 \\
\mathrm{n}^{\circ} \text { pregabalin }=403 \\
\mathrm{n}^{\circ} \text { duloxetine }+ \\
\text { pregabalin }=339 \\
\text { Total } n^{\circ}=1,143\end{array}$ & $514 \mathrm{~F} / 629 \mathrm{M}$ & $61.5 \pm 10.62$ & $60 \mathrm{mg}$ & 20 weeks & BPI-MSF, BDI-II & $P=0.370$ & 0.539 & RCT double-blind & $\begin{array}{l}\text { Efficacy DPNP } \\
\text { BPI-MSF }\end{array}$ \\
\hline Boyle et al. (89) & $\begin{array}{l}\mathrm{n}^{\circ} \text { duloxetine }=28 \\
\mathrm{n}^{\circ} \text { pregabalin }=27 \\
\mathrm{n}^{\circ} \text { amitriptyline }=28 \\
\text { Total } \mathrm{n}^{\circ}=83\end{array}$ & $26 \mathrm{~F} / 57 \mathrm{M}$ & $65.1 \pm 8.9$ & $60-120 \mathrm{mg}$ & 4 weeks & BPI-S, SF-36, PSG & $P<0.05$ & $\begin{array}{l}0.500 \\
1.000\end{array}$ & RCT double-blind & $\begin{array}{l}\text { Impact on pain, } \\
\text { polysomnographic } \\
\text { sleep, daytime } \\
\text { functioning, and } \\
\text { quality of life in DPNP } \\
\text { BPI-S }\end{array}$ \\
\hline $\begin{array}{l}\text { Tanenberg et al. } \\
\text { (90) }\end{array}$ & $\begin{array}{l}\mathrm{n}^{\circ} \text { duloxetine }=138 \\
\mathrm{n}^{\circ} \text { pregabalin }=134 \\
\mathrm{n}^{\circ} \text { duloxetine }+ \\
\text { gabapentin }=135 \\
\text { Total } \mathrm{n}^{\circ}=407\end{array}$ & $165 \mathrm{~F} / 242 \mathrm{M}$ & $60.9 \pm 10.2$ & $60 \mathrm{mg}$ & 12 weeks & $\begin{array}{l}\text { BPI, BDI-II, PGI-I, } \\
\text { SDS, TEAEs, Pain } \\
\text { rating }\end{array}$ & $P=0.08$ & 1.000 & $\begin{array}{l}\text { RCT open-label } \\
\text { non-inferiority study }\end{array}$ & $\begin{array}{l}\text { Duloxetine is } \\
\text { non-inferior to (as } \\
\text { good as) pregabalin in } \\
\text { DPNP } \\
\text { Pain rating }\end{array}$ \\
\hline
\end{tabular}


TABLE 1 | Continued

\begin{tabular}{|c|c|c|c|c|c|c|c|c|c|c|}
\hline References & $\mathbf{N}^{\circ}$ participants & Gender & $\begin{array}{l}\text { Years (mean }+ \\
\text { SD) }\end{array}$ & $\begin{array}{c}\text { Dose } \\
\text { duloxetine }\end{array}$ & $\begin{array}{l}\text { Treatment } \\
\text { duration }\end{array}$ & $\begin{array}{l}\text { Diagnosis scales, } \\
\text { measures }\end{array}$ & $P$-value & G Hedges & Type of study & Outcome \\
\hline $\begin{array}{l}\text { Skljarevski et al. } \\
(91)\end{array}$ & $\begin{array}{l}\mathrm{n}^{\circ} \text { duloxetine } \mathrm{QD}= \\
115 \\
\mathrm{n}^{\circ} \text { duloxetine }=216 \\
\text { Total } \mathrm{n}^{\circ}=331\end{array}$ & $134 \mathrm{~F} / 197 \mathrm{M}$ & $62.6 \pm 9.4$ & $60-120 \mathrm{mg}$ & 26 weeks & Pain rating, $\mathrm{BPI}$ & $P=0.017$ & 2.562 & RCT open-label & $\begin{array}{l}\text { Effect of duloxetine } \\
60 \mathrm{mg} \text { QD in patients } \\
\text { with DPNP } \\
\text { Pain ratings }\end{array}$ \\
\hline $\begin{array}{l}\text { Armstrong et al. } \\
\text { (92) }\end{array}$ & $\begin{array}{l}\mathrm{n}^{\circ} \text { duloxetine } \mathrm{QD}= \\
344 \\
\mathrm{n}^{\circ} \text { duloxetine } \mathrm{BID}= \\
341 \\
\mathrm{n}^{\circ} \text { placebo }=339 \\
\text { Total } \mathrm{n}^{\circ}=1,024\end{array}$ & $572 \mathrm{~F} / 452 \mathrm{M}$ & $59.7 \pm 10.7$ & $\begin{array}{l}60 \mathrm{mg} \text { QD } \\
60 \mathrm{mg} \text { BID }\end{array}$ & 12 weeks & $\begin{array}{l}\text { DSM-IV, MINI, } \\
\text { SF-36, BPI, EQ-5D }\end{array}$ & $\begin{array}{l}P=0.004 \\
P<0.001\end{array}$ & $\begin{array}{l}10.00 \\
10.00\end{array}$ & RCT double-blind & $\begin{array}{l}\text { Efficacy in DPNP } \\
\text { EQ-5D index }\end{array}$ \\
\hline $\begin{array}{l}\text { Wernicke et al. } \\
\text { (93) }\end{array}$ & $\begin{array}{l}\mathrm{n}^{\circ} \text { duloxetine }=197 \\
\mathrm{n}^{\circ} \text { routine care }=96 \\
\text { Total } \mathrm{n}^{\circ}=293\end{array}$ & $158 \mathrm{~F} / 135 \mathrm{M}$ & $58.1 \pm 10.5$ & $120 \mathrm{mg}$ & 52 weeks & $\begin{array}{l}\text { DSM-IV, MNSI, } \\
\text { TEAEs, SF-36, } \\
\text { EQ-5D, TEAES }\end{array}$ & $\begin{array}{c}P<0.01 \\
\text { TEAEs }=5.6 \%\end{array}$ & 4.020 & RCT open-label & $\begin{array}{l}\text { Safety at a fixed-dose } \\
\text { of } 60 \mathrm{mg} \text { BID in DPNP } \\
\text { SF-36 }\end{array}$ \\
\hline Raskin et al. (94) & $\begin{array}{l}\mathrm{n}^{\circ} \text { duloxetine } \mathrm{BID}= \\
334 \\
\mathrm{n}^{\circ} \text { placebo } \mathrm{QD}=115 \\
\text { Total } \mathrm{n}^{\circ}=449\end{array}$ & $215 \mathrm{~F} / 234 \mathrm{M}$ & $59.8 \pm 10.6$ & $\begin{array}{r}120 \mathrm{mg} \text { QD } \\
60 \mathrm{mg} \text { BID }\end{array}$ & 28 weeks & $\begin{array}{l}\text { BPI, CGI-S, MNSI, } \\
\text { TEAES }\end{array}$ & $\begin{array}{c}P=0.020 \\
\text { TEAEs }<5 \%\end{array}$ & 0.229 & RCT open-label & $\begin{array}{l}\text { Safety and tolerability } \\
\text { in diabetic neuropathy } \\
\text { TEAEs }\end{array}$ \\
\hline $\begin{array}{l}\text { Goldstein et al. } \\
\text { (95) }\end{array}$ & $\begin{array}{l}\mathrm{n}^{\circ} \text { duloxetine } 20= \\
115 \\
\mathrm{n}^{\circ} \text { duloxetine } 60= \\
114 \\
\mathrm{n}^{\circ} \text { duloxetine } 120= \\
113 \\
\mathrm{n}^{\circ} \text { placebo }=115 \\
\text { Total } \mathrm{n}^{\circ}=457\end{array}$ & $176 \mathrm{~F} / 281 \mathrm{M}$ & $60.1 \pm 10.9$ & $20-120 \mathrm{mg}$ & 12 weeks & $\begin{array}{l}\text { DSM-IV, MINI, MNSI, } \\
\text { 24-h Average Pain } \\
\text { Score, BPI-S, AEs }\end{array}$ & $\begin{array}{l}P>0.05 \\
P \leq 0.01 \\
P \leq 0.001 \\
A E S<20 \%\end{array}$ & $\begin{array}{l}1.000 \\
3.667 \\
4.791\end{array}$ & RCT double-blind & $\begin{array}{l}\text { Efficacy and safety in } \\
\text { diabetic neuropathy } \\
\text { 24-h Average Pain } \\
\text { Score } \\
\text { BPI-S }\end{array}$ \\
\hline Raskin et al. (96) & $\begin{array}{l}\mathrm{n}^{\circ} \text { duloxetine } \mathrm{QD}= \\
116 \\
\mathrm{n}^{\circ} \text { duloxetine } \mathrm{BID}= \\
116 \\
\mathrm{n}^{\circ} \text { placebo }=116 \\
\text { Total } \mathrm{n}^{\circ}=348\end{array}$ & $186 \mathrm{~F} / 162 \mathrm{M}$ & $58.8 \pm 10.1$ & $60-120 \mathrm{mg}$ & 12 weeks & $\begin{array}{l}\text { DSM-IV, MINI, MNSI, } \\
\text { 24-h Average Pain } \\
\text { Score, TEAEs }\end{array}$ & $\begin{array}{c}P<0.001 \\
\text { TEAEs }=12.1 \%\end{array}$ & $\begin{array}{l}5.000 \\
4.833\end{array}$ & RCT double-blind & $\begin{array}{l}\text { Efficacy and safety in } \\
\text { DPNP } \\
24-h \text { average } \\
\text { pain score }\end{array}$ \\
\hline \multicolumn{11}{|l|}{ Fibromyalgia } \\
\hline $\begin{array}{l}\text { Murakami et al. } \\
(97)\end{array}$ & $\begin{array}{l}n^{\circ} \text { duloxetine }=50 \\
n^{\circ} \text { placebo }=71 \\
\text { Total } n^{\circ}=121\end{array}$ & $99 \mathrm{~F} / 22 \mathrm{M}$ & $47.3 \pm 11.9$ & $20-60 \mathrm{mg}$ & 48 weeks & $\begin{array}{l}\text { BPI, PGI-I, CGI-I, } \\
\text { FIQ, BDI-II, SF-36, } \\
\text { AEs }\end{array}$ & $\begin{array}{c}P<0.05 \\
\text { AEs } 10.1 \% \\
\text { (moderate AE) }\end{array}$ & 0.159 & $\begin{array}{l}\text { RCT open-label, } \\
\text { phase III }\end{array}$ & $\begin{array}{l}\text { Efficacy and safety } \\
\text { BPI }\end{array}$ \\
\hline $\begin{array}{l}\text { Murakami et al. } \\
\text { (98) }\end{array}$ & $\begin{array}{l}\mathrm{n}^{\circ} \text { duloxetine }=191 \\
\mathrm{n}^{\circ} \text { placebo }=195 \\
\text { Total } \mathrm{n}^{\circ}=386\end{array}$ & $321 \mathrm{~F} / 65 \mathrm{M}$ & $48.7 \pm 11.9$ & $60 \mathrm{mg}$ & 14 weeks & $\begin{array}{l}\mathrm{BPI}, \mathrm{FIQ}, \mathrm{SF}-36 \text {, } \\
\mathrm{BDI}-\|\end{array}$ & $P=0.5456$ & 0.061 & $\begin{array}{l}\text { RCT double-blind } \\
\text { phase III }\end{array}$ & BPI change average \\
\hline Mohs et al. (99) & $\begin{array}{l}\mathrm{n}^{\circ} \text { duloxetine }=80 \\
\mathrm{n}^{\circ} \text { placebo }=76 \\
\text { Total } \mathrm{n}^{\circ}=156\end{array}$ & $144 \mathrm{~F} / 12 \mathrm{M}$ & $21-88$ & $60-120 \mathrm{mg}$ & 24 weeks & $\begin{array}{l}\text { BPI, DSM-IV, VLRT, } \\
\text { SDST, TMT }\end{array}$ & $P>0.05$ & 0.065 & RCT double-blind & $\begin{array}{l}\text { Cognition } \\
\text { effectiveness } \\
\text { BPI }\end{array}$ \\
\hline
\end{tabular}


TABLE 1 | Continued

\begin{tabular}{|c|c|c|c|c|c|c|c|c|c|c|}
\hline References & $\mathbf{N}^{\circ}$ participants & Gender & $\begin{array}{l}\text { Years (mean + } \\
\text { SD) }\end{array}$ & $\begin{array}{l}\text { Dose } \\
\text { duloxetine }\end{array}$ & $\begin{array}{l}\text { Treatment } \\
\text { duration }\end{array}$ & $\begin{array}{l}\text { Diagnosis scales, } \\
\text { measures }\end{array}$ & $P$-value & G Hedges & Type of study & Outcome \\
\hline Mease et al. (33) & $\begin{array}{l}\text { Study 1: total }=278 \\
n^{\circ} \text { duloxetine } 120= \\
79 \\
n^{\circ} \text { duloxetine } 60 / 120 \\
=127 \\
n^{\circ} \text { placebo/Dlx }=72 \\
\text { Study 2: total: } 204 \\
n^{\circ} \text { duloxetine } 60= \\
17 \\
n^{\circ} \text { duloxetine } \\
120=82 \\
n^{\circ} \text { duloxetine } 60 / 120 \\
=2 \\
n^{\circ} \\
\text { placebo/duloxetine } \\
60=103\end{array}$ & $\begin{array}{l}\text { Study 1: } 267 \\
\text { F/11 M } \\
\text { Study 2: } \\
194 \text { F/10M }\end{array}$ & $52.0 \pm 9.6$ & $60-120 \mathrm{mg}$ & 28 weeks & $\begin{array}{l}\text { BPI, PGI-I, BDI-II, } \\
\text { HDRS, SF-36 }\end{array}$ & $\begin{array}{l}\text { Study 1: } \\
P<0.001 \\
\text { Study 2: } \\
P=0.580\end{array}$ & $\begin{array}{c}\text { Study 1: } \\
0.297 \\
0.406 \\
\text { Study 2: } \\
0.247 \\
0.055 \\
0.000\end{array}$ & $\begin{array}{l}2 \text { RCT double-blind } \\
\text { phase II }\end{array}$ & $\begin{array}{l}\text { Risk/benefit profile for } \\
\text { duloxetine } \\
\text { BPI }\end{array}$ \\
\hline $\begin{array}{l}\text { Arnold et al. } \\
(100)\end{array}$ & $\begin{array}{l}\mathrm{n}^{\circ} \text { duloxetine }=263 \\
\mathrm{n}^{\circ} \text { placebo }=267 \\
\text { Total } \mathrm{n}^{\circ}=530\end{array}$ & $244 \mathrm{~F} / 19 \mathrm{M}$ & $50.7 \pm 11.3$ & $60-120 \mathrm{mg}$ & 24 weeks & $\begin{array}{l}\text { BPI, CGI-S, BDI, } \\
\text { SF-36, DSM-IV, BAI, } \\
\text { CPFQ, MFI }\end{array}$ & $P<0.001$ & 4.000 & RCT double-blind & $\begin{array}{l}\text { Symptoms } \\
\text { improvement } \\
\text { BPI }\end{array}$ \\
\hline $\begin{array}{l}\text { Chappell et al. } \\
\text { (101) }\end{array}$ & $\begin{array}{l}\mathrm{n}^{\circ} \text { duloxetine } 60= \\
104 \\
\mathrm{n}^{\circ} \text { duloxetine } 120= \\
203 \\
\text { Total } \mathrm{n}^{\circ}=307\end{array}$ & $293 \mathrm{~F} / 14 \mathrm{M}$ & $49.0 \pm 11.07$ & $60-120 \mathrm{mg}$ & 52 weeks & $\begin{array}{l}\text { BPI, FIQ, PGI-I, } \\
\text { CGI-S, SDS, AES }\end{array}$ & $\begin{array}{c}P \leq 0.05 \\
\mathrm{AEs}=21.1 \%\end{array}$ & 1.843 & $\begin{array}{l}\text { RCT double-blind } \\
\text { phase III }\end{array}$ & $\begin{array}{l}\text { Efficacy and safety } \\
\text { FIQ }\end{array}$ \\
\hline $\begin{array}{l}\text { Russell et al. } \\
\text { (102) }\end{array}$ & $\begin{array}{l}\mathrm{n}^{\circ} \text { duloxetine }=376 \\
\mathrm{n}^{\circ} \text { placebo }=144 \\
\text { Total } \mathrm{n}^{\circ}=520\end{array}$ & $356 \mathrm{~F} / 20 \mathrm{M}$ & $51.3 \pm 10.9$ & 20-120mg & 24 weeks & BPI, PGI-I, AEs & $\begin{array}{c}P \leq 0.05 \\
A E s=18.0 \%\end{array}$ & $\begin{array}{l}3.953 \\
2.619\end{array}$ & RCT double-blind & $\begin{array}{l}\text { Efficacy and safety } \\
\text { BPI }\end{array}$ \\
\hline $\begin{array}{l}\text { Arnold et al. } \\
\text { (103) }\end{array}$ & $\begin{array}{l}\mathrm{n}^{\circ} \text { duloxetine }=240 \\
\mathrm{n}^{\circ} \text { placebo }=118 \\
\text { Total } \mathrm{n}^{\circ}=358\end{array}$ & $240 \mathrm{~F}$ & $49.6 \pm 10.9$ & $60-120 \mathrm{mg}$ & 12 weeks & $\begin{array}{l}\text { BPI, FIQ, CGI-S, } \\
\text { PGI-I, HDRS, QLDS, } \\
\text { SF-36, SDS, TEAEs }\end{array}$ & $\begin{array}{c}P<0.01 \\
\text { TEAEs }=18.7 \%\end{array}$ & $\begin{array}{l}5.721 \\
5.768\end{array}$ & RCT double-blind & $\begin{array}{l}\text { Efficacy and safety } \\
\text { BPI }\end{array}$ \\
\hline $\begin{array}{l}\text { Arnold et al. } \\
\text { (104) }\end{array}$ & $\begin{array}{l}\mathrm{n}^{\circ} \text { duloxetine }=104 \\
\mathrm{n}^{\circ} \text { placebo }=103 \\
\text { Total } \mathrm{n}^{\circ}=207\end{array}$ & $92 \mathrm{~F} / 12 \mathrm{M}$ & $49.9 \pm 12.3$ & $120 \mathrm{mg}$ & 12 weeks & $\begin{array}{l}\text { FIQ, CGI-S, PGI-I, } \\
\text { DSM-IV, BDI-II, BAI, } \\
\text { SF-36, QLDS, SDS, } \\
\text { TEAES }\end{array}$ & $\begin{array}{c}P=0.027 \\
P_{\text {TEAEs }}=0.229\end{array}$ & 3.115 & RCT double-blind & $\begin{array}{l}\text { Efficacy and safety } \\
\text { FIQ }\end{array}$ \\
\hline \multicolumn{11}{|c|}{ Stress urinary incontinence } \\
\hline $\begin{array}{l}\text { Cornu et al. } \\
(105)\end{array}$ & $\begin{array}{l}\mathrm{n}^{\circ} \text { duloxetine }=16 \\
\mathrm{n}^{\circ} \text { placebo }=15 \\
\text { Total } \mathrm{n}^{\circ}=31\end{array}$ & $31 \mathrm{M}$ & $68.3 \pm 6.9$ & $80 \mathrm{mg}$ & 12 weeks & $\begin{array}{l}\text { I-QoL, IIQ-SF, } \\
\text { UDI-SF, USPQ, } \\
\text { ICIQ-SF, BDI-II, IEF, } \\
\text { TEAEs }\end{array}$ & $\begin{array}{c}P<0.0001 \\
P_{\text {TEAES }}=0.27\end{array}$ & 1.735 & RCT double-blind & $\begin{array}{l}\text { Efficacy and safety } \\
\text { IEF }\end{array}$ \\
\hline $\begin{array}{l}\text { Cardozo et al. } \\
\text { (106) }\end{array}$ & $\begin{array}{l}\mathrm{n}^{\circ} \text { duloxetine }= \\
1,378 \\
\mathrm{n}^{\circ} \text { placebo }=1,380 \\
\text { Total } \mathrm{n}^{\circ}=2,758\end{array}$ & $2,758 \mathrm{~F}$ & $55.51 \pm 11.77$ & $80 \mathrm{mg}$ & 6 weeks & $\begin{array}{l}\text { IEF, PGIII, KHQ, } \\
\text { TEAEs }\end{array}$ & $\begin{array}{c}P<0.001 \\
\text { TEAEs }=21 \%\end{array}$ & 0.235 & RCT double-blind & $\begin{array}{l}\text { Efficacy and safety } \\
\text { IEF }\end{array}$ \\
\hline
\end{tabular}


TABLE 1 | Continued

\begin{tabular}{|c|c|c|c|c|c|c|c|c|c|c|}
\hline References & $\mathbf{N}^{\circ}$ participants & Gender & $\begin{array}{l}\text { Years (mean + } \\
\text { SD) }\end{array}$ & $\begin{array}{c}\text { Dose } \\
\text { duloxetine }\end{array}$ & $\begin{array}{l}\text { Treatment } \\
\text { duration }\end{array}$ & $\begin{array}{l}\text { Diagnosis scales, } \\
\text { measures }\end{array}$ & $P$-value & G Hedges & Type of study & Outcome \\
\hline Bent et al. (107) & $\begin{array}{l}\mathrm{n}^{\circ} \text { duloxetine }=300 \\
\mathrm{n}^{\circ} \text { placebo }=288 \\
\text { Total } \mathrm{n}^{\circ}=588\end{array}$ & $588 \mathrm{~F}$ & $53.2 \pm 12.5$ & $80 \mathrm{mg}$ & 8 weeks & $\begin{array}{l}\text { IEF, ICIQ-SF, I-QOL, } \\
\text { PGI-I, TEAES }\end{array}$ & $\begin{array}{c}P<0.001 \\
\text { TEAEs }=21.3 \%\end{array}$ & - & RCT double-blind & $\begin{array}{l}\text { Efficacy and safety } \\
\text { IEF }\end{array}$ \\
\hline Lin et al. (108) & $\begin{array}{l}\mathrm{n}^{\circ} \text { duloxetine }=61 \\
\mathrm{n}^{\circ} \text { placebo }=60 \\
\text { Total } \mathrm{n}^{\circ}=121\end{array}$ & $121 \mathrm{~F}$ & $56.31 \pm 11.0$ & $80 \mathrm{mg}$ & 8 weeks & $\begin{array}{l}\text { IEF, I-QOL, PGI-I, } \\
\text { TEAEs }\end{array}$ & $\begin{array}{c}P<0.001 \\
\text { TEAEs }=26.7 \%\end{array}$ & - & RCT double-blind & $\begin{array}{l}\text { Efficacy and safety } \\
\text { IEF }\end{array}$ \\
\hline $\begin{array}{l}\text { Schagen et al. } \\
\text { (109) }\end{array}$ & $\begin{array}{l}\mathrm{n}^{\circ} \text { duloxetine }=131 \\
\mathrm{n}^{\circ} \text { placebo }=134 \\
\text { Total } \mathrm{n}^{\circ}=165\end{array}$ & $165 \mathrm{~F}$ & $70.63 \pm 5.08$ & $40-80 \mathrm{mg}$ & 12 weeks & $\begin{array}{l}\text { IEF, PGI-I, I-QOL, } \\
\text { BDI-II, AEs }\end{array}$ & $\begin{array}{c}P<0.001 \\
\text { PTEAES }=0.210\end{array}$ & - & $\begin{array}{l}\text { RCT double-blind } \\
\text { phase IV }\end{array}$ & $\begin{array}{l}\text { Efficacy and safety in } \\
\text { community-dwelling } \\
\text { women } \geq 65 \text { years } \\
\text { IEF }\end{array}$ \\
\hline $\begin{array}{l}\text { Castro-Diaz } \\
\text { et al. (110) }\end{array}$ & $\begin{array}{l}\mathrm{n}^{\circ} \text { duloxetine } 20 \mathrm{BID} \\
=133 \\
\mathrm{n}^{\circ} \text { duloxetine } 40 \mathrm{QD} \\
=127 \\
\mathrm{n}^{\circ} \text { duloxetine } 40 \mathrm{BID} \\
=136 \\
\mathrm{n}^{\circ} \text { placebo }=120 \\
\text { Total } \mathrm{n}^{\circ}=516\end{array}$ & $516 \mathrm{~F}$ & $53.0 \pm 10.6$ & $20-80 \mathrm{mg}$ & 8 weeks & $\begin{array}{l}\text { IEF, ICIQ-SF, I-QOL, } \\
\text { PGI-I, TEAES }\end{array}$ & $\begin{array}{c}P=0.008 \\
\text { TEAEs }=11.8 \%\end{array}$ & $\begin{array}{l}0.331 \\
0.436 \\
0.611\end{array}$ & RCT double-blind & $\begin{array}{l}\text { Effect of dose } \\
\text { escalation on the } \\
\text { tolerability and efficacy } \\
\text { TEAEs }\end{array}$ \\
\hline $\begin{array}{l}\text { Schlenker et al. } \\
\text { (111) }\end{array}$ & $\begin{array}{l}\mathrm{n}^{\circ} \text { duloxetine } \mathrm{BID}= \\
20 \\
\text { Total } \mathrm{n}^{\circ}=20\end{array}$ & $20 \mathrm{M}$ & 65.6 & $80 \mathrm{mg}$ & 1-35 weeks & SUIQ, AES & $\begin{array}{c}P<0.001 \\
\text { TEAEs }=30 \%\end{array}$ & 0.655 & Cohort study & $\begin{array}{l}\text { Efficacy and safety for } \\
\text { men with stress } \\
\text { incontinence (use } \\
\text { off-label) } \\
\text { Average daily use of } \\
\text { incontinence pads }\end{array}$ \\
\hline $\begin{array}{l}\text { Weinstein et al. } \\
(112)\end{array}$ & $\begin{array}{l}\mathrm{n}^{\circ} \text { duloxetine } \mathrm{BID}= \\
2,960 \\
\text { Total } \mathrm{n}^{\circ}=2,960\end{array}$ & $2,960 \mathrm{~F}$ & 49.6 & $80 \mathrm{mg}$ & 10 weeks & $\begin{array}{l}\text { SUIQ, I-QOL, PGI-S, } \\
\text { BDI-II, IEF, AEs }\end{array}$ & $\begin{array}{c}P<0.05 \\
\text { AEs }=24.3 \%\end{array}$ & $\begin{array}{c}0.186 \\
0.113 \\
\text { Comparation } \\
\text { with } \\
\text { Caucasian subgro }\end{array}$ & $\begin{array}{l}\text { RCT open-label } \\
\text { phase III } \\
\text { up }\end{array}$ & $\begin{array}{l}\text { Efficacy and safety in } \\
\text { racial and ethnic } \\
\text { subgroups } \\
\text { I-QOL }\end{array}$ \\
\hline $\begin{array}{l}\text { Ghoniem et al. } \\
\text { (113) }\end{array}$ & $\begin{array}{l}\mathrm{n}^{\circ} \text { duloxetine }=52 \\
\mathrm{n}^{\circ} \mathrm{PFMT}=50 \\
\mathrm{n}^{\circ} \text { no PFMT }=47 \\
\mathrm{n}^{\circ} \text { combined }=52 \\
\text { Total } \mathrm{n}^{\circ}=201\end{array}$ & $201 \mathrm{~F}$ & 53 & $80 \mathrm{mg}$ & 12 weeks & $\begin{array}{l}\text { SUI, IEF, I-QOL, } \\
\text { PGI-I }\end{array}$ & $P<0.001$ & 0.043 & RCT double-blind & $\begin{array}{l}\text { Efficacy of duloxetine } \\
\text { alone or combined } \\
\text { with PFMT } \\
\text { IEF }\end{array}$ \\
\hline $\begin{array}{l}\text { Kinchen et al. } \\
\text { (114) }\end{array}$ & $\begin{array}{l}\mathrm{n}^{\circ} \text { duloxetine } \mathrm{BID}= \\
224 \\
\mathrm{n}^{\circ} \text { placebo }=227 \\
\text { Total } \mathrm{n}^{\circ}=451\end{array}$ & $451 \mathrm{~F}$ & $52.7 \pm 13.0$ & $80 \mathrm{mg}$ & 12 weeks & I-QOL, PGI-I & $P=0.07$ & - & RCT double-blind & $\begin{array}{l}\text { Effectiveness in } \\
\text { improving quality of life } \\
\text { I-QOL }\end{array}$ \\
\hline $\begin{array}{l}\text { Cardozo et al. } \\
\text { (115) }\end{array}$ & $\begin{array}{l}\mathrm{n}^{\circ} \text { duloxetine }=55 \\
\mathrm{n}^{\circ} \text { placebo }=54 \\
\text { Total } \mathrm{n}^{\circ}=109\end{array}$ & $109 \mathrm{~F}$ & $54.5 \pm 9.7$ & $80-120 \mathrm{mg}$ & 8 weeks & I-QOL, IEF & $P=0.003$ & 0.545 & RCT double-blind & $\begin{array}{l}\text { Efficacy } \\
\text { I-QOL }\end{array}$ \\
\hline
\end{tabular}


TABLE 1 | Continued

\begin{tabular}{|c|c|c|c|c|c|c|c|c|c|c|}
\hline References & $\mathbf{N}^{\circ}$ participants & Gender & $\begin{array}{l}\text { Years (mean + } \\
\text { SD) }\end{array}$ & $\begin{array}{c}\text { Dose } \\
\text { duloxetine }\end{array}$ & $\begin{array}{l}\text { Treatment } \\
\text { duration }\end{array}$ & $\begin{array}{l}\text { Diagnosis scales, } \\
\text { measures }\end{array}$ & $P$-value & G Hedges & Type of study & Outcome \\
\hline $\begin{array}{l}\text { Millard et al. } \\
\text { (116) }\end{array}$ & $\begin{array}{l}\mathrm{n}^{\circ} \text { duloxetine } \mathrm{BID}= \\
227 \\
\mathrm{n}^{\circ} \text { placebo }=231 \\
\text { Total } \mathrm{n}^{\circ}=458\end{array}$ & $458 \mathrm{~F}$ & 53.7 & $80 \mathrm{mg}$ & 12 weeks & $\begin{array}{l}\text { SUI, IEF, I-QOL, } \\
\text { PGI-I, PGI-S, AEs }\end{array}$ & $\begin{array}{c}P=0.007 \\
A E s=17.2 \%\end{array}$ & 0.236 & RCT double-blind & $\begin{array}{l}\text { Efficacy and safety } \\
\text { I-QOL }\end{array}$ \\
\hline $\begin{array}{l}\text { Van Kerrebroeck } \\
\text { et al. (117) }\end{array}$ & $\begin{array}{l}\mathrm{n}^{\circ} \text { duloxetine } \mathrm{BID}= \\
247 \\
\mathrm{n}^{\circ} \text { placebo }=247 \\
\text { Total } \mathrm{n}^{\circ}=494\end{array}$ & $494 \mathrm{~F}$ & $52.0 \pm 11$ & $80 \mathrm{mg}$ & 12 weeks & $\begin{array}{l}\text { IEF, I-QOL, PGI-I, } \\
\text { PGI-S, TEAEs }\end{array}$ & $\begin{array}{c}P=0.008 \\
\text { TEAEs }=22 \%\end{array}$ & - & RCT double-blind & $\begin{array}{l}\text { Efficacy and safety } \\
\text { I-QOL }\end{array}$ \\
\hline $\begin{array}{l}\text { Dmochowski } \\
\text { et al. (118) }\end{array}$ & $\begin{array}{l}\mathrm{n}^{\circ} \text { duloxetine } \mathrm{BID}= \\
344 \\
\mathrm{n}^{\circ} \text { placebo }=339 \\
\text { Total } \mathrm{n}^{\circ}=683\end{array}$ & $683 \mathrm{~F}$ & $52.3 \pm 10.4$ & $80 \mathrm{mg}$ & 12 weeks & $\begin{array}{l}\text { SUI, IEF, I-QOL, } \\
\text { PGI-I, BDI-II, TEAEs }\end{array}$ & $\begin{array}{c}P<0.001 \\
\text { TEAEs }=24 \%\end{array}$ & 0.332 & RCT double-blind & $\begin{array}{l}\text { Efficacy and safety } \\
\text { IEF, I-QOL }\end{array}$ \\
\hline
\end{tabular}

MDD, Major Depressive Disorder; DSM-IV, Diagnostic and Statistical Manual of Mental Disorders; HAMD, Hamilton Rating Scale for Depression; SCID, Structured Clinical Interview for DSM; CGI-I, Clinical Impressions of Improvement; RCT, Randomized Controlled Trial; GDS, Geriatric Depression Scale; CGI-S, Clinical Global Impression of Severity; PGI-I, Patient's Global Impressions of Improvement; BPI, Brief Pain Inventory; NRS, Numeric Rating Scale; TEAES, Treatment-emergent adverse events; QD, Once Daily; BID, Twice Daily; QIDS-SR, Quick Inventory of Depressive Symptomatology-Self-Rated; SDS, Sheehan Disability Scale; SSRIs, Selective Serotonin Reuptake Inhibitors; SASS, Social Adaptation Self-evaluation Scale; GAF, Global Assessment of Functioning Scale; MADRS, Montgomery-Asberg Depression Rating Scale; HDRS, Hamilton Depression Rating Scale; MINI, Mini International Neuropsychiatric Interview; BPI-SF, Brief Pain Inventory -Short Form; WAIS, Wechsler Adult Intelligence Scale; SWM, Spatial Working Memory; RVIP, Rapid Visual Information Processing; MTS, Match to Sample Visual Search; ID/ED, Intra-Extra-Dimensional Set Shift: SOC, Stockings of Cambridge; BDI, Beck Depression Inventory: VAS, Visual Analog Scale: AEs, Adverse Events: SF-36, Medical Outcomes Study 36-item short-form health survey: SQ-SS, Symptom Questionnaire, Somatic Subscale: MMSE, Mini-Mental State Examination; UKU, Udvalg for Kliniske Undersogelser-Committee of Clinical Investigations Side Effect Rating Scale; IDS-C-30, 30- item Inventory of Depressive Symptomatology-Clinician Rated; EQ-5D, European QOL Questionnaire-5 Dimension; HAMA, Hamilton Anxiety Rating Scale; WHOQLL-BREF. World Health Organization Quality of Life Scale; CSFQ, Changes in Sexual Functioning Questionnaire; CCS, Composite Cognitive Score; QLDS, Quality of Life in Depression Scale; HADS, Hospital Anxiety and Depression Scale; CAS, Covi Anxiety Scale; RDS, Raskin Depression Scale; SIGH-A, Structured Clinical Interview Guide for Hamilton Anxiety Cognitive Score; QLDS, Quality of Life in Depression Scale; HADS, Hospital Anxiety and Depression Scale; CAS, Covi Anxiety Scale; RDS, Raskin Depression Scale; SIGH-A, Structured Clinical Interview Guide for Hamilton Anxiety
Rating Scale; NCI-CTCAE v4.03, National Cancer Institute Common Terminology Criteria for Adverse Events version 4.03; PNQ, Patient Neurotoxicity Questionnaire; SF-MPQ, Short form of the McGill Pain Questionnaire; McGill, Pain Questionnaire; DN-4, Neuropathic Pain Diagnostic Questionnaire; RTOG, Radiation Therapy Oncology Group; C-SSRS, Columbia Suicide Severity Rating Scale; QOL, Quality of Life; LSEQ, Leeds Sleep Evaluation Questionnaire; DPNP, Diabetic Peripheral Neuropathic Pain; API, Average Pain Intensity; MS-QoL-54, Multiple Sclerosis Quality of Life-54 Instrument; MFIS, Modified Fatigue Impact Scale; BPI-MSF, Brief Pain Inventory Modified Short Form; PSG, Polysomnographic; MNSI, Michigan Neuropathy Screening Instrument; FIQ, Fibromyalgia Impact Questionnaire; VLRT, Verbal Learning and Recall Test; SDST, Symbol Digit Substitution Test; TMT, Trail-Making Test; BAI, Beck Anxiety Inventory; CPFQ, Cognitive and Physical Functioning Questionnaire; MFI, Multidimensional Fatigue Inventory; I-QoL, Incontinence Quality of Life; PFMT, Pelvic Floor Muscle Training; IIQ-SF, Incontinence Impact Questionnaire Short Form; UDI-SF, Urogenital Distress Inventory Short Form; USPQ, Urinary Symptom Profile Questionnaire; ICIQ-UI-SF, Incontinence Questionnaire-Urinary Incontinence Short Form; IEF, Incontinence Episode Frequency; KHQ, King's Health Questionnaire; SUI, Stress Urinary Incontinence; SUIQ, Stress/Urge Incontinence Questionnaire. 


\section{DISCUSSION}

In the last years, mutual pathophysiological mechanisms have been identified in depression, pain, and anxiety (11). Neuropathic pain, specifically DPNP, coexists with mental disorders, predominantly with depression and anxiety (123). Highlighting the functional impairment as a result of unremitting pain symptoms, neuropathy has been correlated with an increased risk of depression (124). On the other hand, the widespread spontaneous pain is the most debilitating symptom of FMS, that might be a link to depression and anxiety disorders as comorbid conditions (125). Due to the similar pathophysiologic mechanisms and high occurrence of FMS and depression, these clinical conditions were considered under a common approach to affective disorders, GAD and PTSD (19). Urinary incontinence is also a severe problem that affects $15-30 \%$ of adults over 60 years, and several studies have reported a link between urinary incontinence, anxiety and depression in women $(22,126)$.

The core of the pathophysiology of these clinical conditions is mostly due to the disruption of 5-HT and norepinephrine (NE) pathways $(19,22,127)$. The monoaminergic hypothesis is based on a partial or total deficit of 5-HT or NE in the central nervous system (CNS) $(128,129)$. Somatic symptoms such as muscle tension, neuropathic and musculoskeletal pain, fatigue, or dizziness are common in MDD and GAD among other psychiatric disorders as result of aberrant 5-HT and NE neurotransmission (130). Regarding pain, antinociception and pronociceptive modulation occurs through 5-HT receptors, in both the central and peripheral nervous systems (131). As in pain, SUI involves the action of monoaminergic system. Evidence demonstrates that endogenous regulation of serotoninergic and noradrenergic mechanism in the spinal cord works simultaneously to maintain the reflex of urinary continence (132).

Therefore, the pharmacological treatment of clinical conditions with similar pathophysiology involves a global perception of coexisting disorders. In this sense, antidepressants such as duloxetine have been considered effective in the treatment of MDD, GAD, NP, FMS, and SUI (133). Duloxetine is a serotonin-norepinephrine reuptake inhibitor, that is, a potent inhibitor of 5-HT transporter (SERT) and norepinephrine transporter (NET) (134). Due to this dual mechanism, its profile seems to have a different response compared to selective 5-HT reuptake inhibitors (SSRIs) (135). In vivo studies, duloxetine presented preferential inhibition of 5-HT reuptake and low affinity for hitamine-H1, alpha-1-norepinephrine, $5-\mathrm{HT}_{(1 \mathrm{~A}, 1 \mathrm{~B}, 1 \mathrm{D})}$, muscarinic acetylcholine, and opioid receptors (136). Clinically, duloxetine has been approved for diverse clinical conditions, acquiring new evidence over the years, also being prescribed to treat other neuropathic pain conditions and chronic musculoskeletal pain $(80,137)$.

In this systematic review, we considered the efficacy, safety, and tolerability of duloxetine in the treatment of current approved clinical conditions. Firstly, an individual search by clinical condition was achieved based on specific inclusion criteria. This strategy allowed us to find consistent results and objectively evaluate the outcomes. Concerning efficacy, duloxetine demonstrated effectiveness in over $80 \%$ of cases. However, some TEAEs are frequent, such as dry mouth, somnolence, nausea, constipation or hyperhidrosis, tending to decrease in time and disappear with continuing duloxetine therapy. Cardiovascular adverse events, such as hypertension, increased heart rate, myocardial ischemia, are also associated with duloxetine administration (29). Within these, only the increase in heart rate was statistically significant, although not being clinically relevant. In sum, duloxetine was considered in all assessed reports as a safe and well-tolerated treatment even in cardiovascular disease, as well as in elderly patients $(29,51,82,93)$. In this sense, our results prove that duloxetine is an option with a valid and consolidated therapeutic value. Secondly, we focused on the clinical conditions' comorbidity. The coexistence of depression, anxiety, and pain is a frequent state, as well as, depression and SUI, and FMS and depression. Therefore, the treatment with duloxetine is largely used due to its dual mechanism that ameliorate the symptoms associated with the concomitant clinical conditions (e.g., MDD and NP). Moreover, we observed a strong link between MDD and pain. This correlation suggests a bidirectional pattern: MDD could be a predictor of chronic pain which in turn might predict the persistence of MDD (138). Thirdly, although the dropout rate with duloxetine treatment reaches around $20 \%$ in certain cases, similar rates were found in placebo and other antidepressants or anticonvulsants treatments. Finally, some considerations should be taken into account regarding to duloxetine prescription and titration. Alcohol, tobacco and coffee (caffeine) are the most widely consumed psychoactive substances worldwide $(139,140)$. Evidence suggests that plasmatic serum levels of duloxetine were decreased (about 15\%) in smoking patients due to the induction of CYP1A2 (141). Hepatotoxicity was also observed in patients whose alcohol consumption was significant (142). Lastly, caffeine is also metabolized by CYP1A2, like duloxetine, and this may increase duloxetine serum levels. However, this interference needs more supporting evidence.

We performed this systematic review in order to include as much evidence as possible. In this process, we analyzed a large number of studies to support our conclusions. Nevertheless, we found some limitations. The inclusion criteria exclude reports in a language other than English. Thus, significant studies may have been missed with this strategy. Our research protocol dismisses qualitative and pooled integrative data analysis of RCTs to avoid repeated analysis of RCTs data and qualitative findings duplication. Regarding the effect size of studies, some of them did not report sufficient statistical data to compute Hedges's g (e.g., standard deviation). However, we decided to include these 19 studies due to their relevance respect to the evaluated outcomes.

In conclusion, there is a substantial amount of evidence in support of efficacy, safety, and tolerability of duloxetine in the treatment of MDD, GAD, NP, FMS, and SUI. The dose range of $60-120 \mathrm{mg}$ daily demonstrated efficacy in most of the studies assessed. TEAEs were mild to moderate, and AEs decreased or remitted with continuing duloxetine treatment. Treatment discontinuation due to both AEs and ineffectiveness of duloxetine yielded enough acceptable results to conclude that duloxetine is safe and well-tolerated. In addition, duloxetine is a 
monotherapy approach that might be useful to treat concomitant disorders with parallel pathophysiological pathways such as MDD and NP, which is an advantage for patients (avoiding polytherapy issues) and a successful cost-effective alternative.

\section{DATA AVAILABILITY STATEMENT}

All datasets generated for this study are included in the article/Supplementary Material.

\section{AUTHOR CONTRIBUTIONS}

Data analysis and the first draft of the manuscript was written by DR-A and TR-B. All authors contributed to the study design, acquired data to analysis, and read and approved the final manuscript.

\section{FUNDING}

This work was financially backed by the Foundation for Science and Technology (FCT, Fundação para a Ciência e Tecnologia) within the framework of grant SFRH/BD/135623/2018 awarded

\section{REFERENCES}

1. Goldberg DS, McGee SJ. Pain as a global public health priority. BMC Public Health. (2011) 11:1-5. doi: 10.1186/1471-2458-11-770

2. Jacob KS. Depression: a major public health problem in need of a multisectoral response. Indian J Med Res. (2012) 136:537-9.

3. McLaughlin KA. The public health impact of major depression: a call for interdisciplinary prevention efforts. Prev Sci. (2011) 12:361-71. doi: 10.1007/s11121-011-0231-8

4. Kraus C, Kadriu B, Lanzenberger R, Zarate CA, Kasper S. Prognosis and improved outcomes in major depression: a review. Transl Psychiatry. (2019) 9:127. doi: 10.1038/s41398-019-0460-3

5. Sheehan DV. Depression: underdiagnosed, undertreated, underappreciated. Manag Care. (2004) 13:6-8.

6. Trivedi $\mathrm{MH}$, Rush AJ, Wisniewski SR, Nierenberg AA, Warden D, Ritz L, et al. Evaluation of outcomes with citalopram for depression using measurement-based care in STAR* D: implications for clinical practice. Am J Psychiatry. (2006) 163:28-40. doi: 10.1176/appi.ajp.163.1.28

7. Wittchen H, Kessler RC, Beesdo KK, Krause P, Höfler M, Hoyer J. Generalized anxiety and depression in primary care: prevalence, recognition, and management. J Clin Psychiatry. (2002) 63:24-34.

8. Roberge P, Normand-Lauzière F, Raymond I, Luc M, Tanguay-Bernard MM, Duhoux A, et al. Generalized anxiety disorder in primary care: mental health services use and treatment adequacy. BMC Fam Pract. (2015) 16:146. doi: 10.1186/s12875-015-0358-y

9. Tyrer P, Baldwin D. Generalised anxiety disorder. Lancet. (2006) 368:215666. doi: 10.1016/S0140-6736(06)69865-6

10. Katzman MA, Bleau P, Blier P, Chokka P, Kjernisted K, Van Ameringen M, et al. Canadian clinical practice guidelines for the management of anxiety, posttraumatic stress and obsessive-compulsive disorders. BMC Psychiatry. (2014) 14:1-83. doi: 10.1186/1471-244X-14-S1-S1

11. Sheng J, Liu S, Wang Y, Cui R, Zhang X. The link between depression and chronic pain: neural mechanisms in the brain. Neural Plast. (2017) 2017:1-10. doi: 10.1155/2017/9724371

12. Nicholson B, Verma S. Comorbidities in chronic neuropathic pain. Pain Med. (2004) 5:S9-27. doi: 10.1111/j.1526-4637.2004.04019.x

13. Fiore NT, Austin PJ. Are the emergence of affective disturbances in neuropathic pain states contingent on supraspinal neuroinflammation? to DR-A and another grant of Fundación Tatiana Pérez de Guzmanel Bueno provided to CS. Our research was further supported by the Carlos III Health Institute (ISCIII, Instituto Carlos III) through grant P16/00405 and co-funding awarded by the Spanish Foundation of Rare Diseases (FEDER, Fundación Española de Enfermedades Raras) to JO.

\section{ACKNOWLEDGMENTS}

The authors would like to thank the Galicia Sur Health Research Institute (Instituto de Investigación Sanitaria Galicia Sur) and the University Hospital Complex of Vigo (Complexo Hospitalario de Vigo) for their support. In addition, the authors are especially thankful for the aid provided by the Psychiatric Nursing and Psychiatry Services of the Álvaro Cunqueiro and Nicolás Peña hospitals.

\section{SUPPLEMENTARY MATERIAL}

The Supplementary Material for this article can be found online at: https://www.frontiersin.org/articles/10.3389/fpsyt. 2020.554899/full\#supplementary-material

Brain Behav Immun. (2016) 56:397-411. doi: 10.1016/j.bbi.2016. 04.012

14. Torta R, Ieraci V, Zizzi F. A review of the emotional aspects of neuropathic pain: from comorbidity to co-pathogenesis. Pain Ther. (2017) 6:11-7. doi: 10.1007/s40122-017-0088-z

15. Li MJ, Liu LY, Chen L, Cai J, Wan Y, Xing GG. Chronic stress exacerbates neuropathic pain via the integration of stress-Affect-related information with nociceptive information in the central nucleus of the amygdala. Pain. (2017) 158:717-39. doi: 10.1097/j.pain.0000000000000827

16. Arnold LM, Choy E, Clauw DJ, Goldenberg DL, Harris RE, Helfenstein $\mathrm{M}$, et al. Fibromyalgia and chronic pain syndromes: a white paper detailing current challenges in the field. Clin J Pain. (2016) 32:737-46. doi: 10.1097/AJP.0000000000000354

17. Tan AC, Jaaniste T, Champion D. Chronic widespread pain and fibromyalgia syndrome: life-course risk markers in young people. Pain Res Manag. (2019) 2019:1-13. doi: 10.1155/2019/6584753

18. De Roa P, Paris P, Poindessous JL, Maillet O, Héron A. Subjective experiences and sensitivities in women with fibromyalgia: a quantitative and comparative study. Pain Res Manag. (2018) 2018:1-8. doi: 10.1155/2018/82 69564

19. Gracely RH, Ceko M, Bushnell MC. Fibromyalgia and depression. Pain Res Treat. (2012) 2012:1-9. doi: 10.1155/2012/486590

20. Thorlacius S, Stefánsson SB, Ranavaya MI, Walker R. Fibromyalgia and anxiety disorder. Laeknabladid. (2002) 88:815-8.

21. Cardinal TM, Antunes LC, Brietzke AP, Parizotti CS, Carvalho F, De Souza A, et al. Differential neuroplastic changes in fibromyalgia and depression indexed by up-regulation of motor cortex inhibition and disinhibition of the descending pain system: an exploratory study. Front Hum Neurosci. (2019) 13:138. doi: 10.3389/fnhum.2019. 00138

22. Felde G, Ebbesen MH, Hunskaar S. Anxiety and depression associated with urinary incontinence. a 10-year follow-up study from the Norwegian HUNT study (EPINCONT). Neurourol Urodyn. (2017) 36:322-8. doi: 10.1002/nau.22921

23. Rogers RG. Urinary stress incontinence in women. $N$ Engl J Med. (2008) 358:1029-36. doi: 10.1056/NEJMcp0707023

24. Jost WH, Marsalek P. Duloxetine in the treatment of stress urinary incontinence. Ther Clin Risk Manag. (2005) 1:259-64. 
25. Goldstein DJ. Duloxetine in the treatment of major depressive disorder. Neuropsychiatr Dis Treat. (2007) 3:193-209. doi: 10.2147/nedt.2007.3.2.193

26. Scholz BA, Hammonds CL, Boomershine CS. Duloxetine for the management of fibromyalgia syndrome. J Pain Res. (2009) 2:99-108. doi: 10.2147/JPR. S4462

27. Wright A, VanDenBerg C. Duloxetine in the treatment of generalized anxiety disorder. Int J Gen Med. (2009) 2:153-62. doi: 10.2147/IJGM.S4492

28. Iqbal Z, Azmi S, Yadav R, Ferdousi M, Kumar M, Cuthbertson DJ, et al. Diabetic peripheral neuropathy: epidemiology, diagnosis, and pharmacotherapy. Clin Ther. (2018) 40:828-49. doi: 10.1016/j.clinthera.2018.04.001

29. Park K, Kim S, Ko YJ, Park BJ. Duloxetine and cardiovascular adverse events: a systematic review and meta-analysis. J Psychiatr Res. (2020) 124:109-14. doi: 10.1016/j.jpsychires.2020.02.022

30. Nelson JC, Pritchett YL, Martynov O, Yu JY, Mallinckrodt CH, Detke MJ. The safety and tolerability of duloxetine compared with paroxetine and placebo: a pooled analysis of 4 clinical trials. Prim Care Companion J Clin Psychiatry. (2006) 8:212-9. doi: 10.4088/PCC.v08n0404

31. Arnold LM. Duloxetine and other antidepressants in the treatment of patients with fibromyalgia. Pain Med. (2007) 8:S63-74. doi: 10.1111/j.1526-4637.2006.00178.x

32. Carter NJ, McCormack PL. Duloxetine: a review of its use in the treatment of generalized anxiety disorder. CNS Drugs. (2009) 23:523-41. doi: 10.2165/00023210-200923060-00006

33. Mease PJ, Russell IJ, Kajdasz DK, Wiltse CG, Detke MJ, Wohlreich $\mathrm{MM}$, et al. Long-term safety, tolerability, and efficacy of duloxetine in the treatment of fibromyalgia. Semin Arthritis Rheum. (2010) 39:454-64. doi: 10.1016/j.semarthrit.2008.11.001

34. Liberati A, Altman DG, Tetzlaff J, Mulrow C, Gøtzsche PC, Ioannidis JPA, et al. The PRISMA statement for reporting systematic reviews and meta-analyses of studies that evaluate health care interventions: explanation and elaboration. J Clin Epidemiol. (2009) 62:e1-34. doi: 10.1016/j.jclinepi.2009.06.006

35. Berk M, Plessis AD, Birkett M, Richard D, Group LDDS. An openlabel study of duloxetine hydrochloride, a mixed sertotonin and noradrenaline reuptake inhibitor, in patients with DSM-III-R major depressive disorder. Int Clin Psychopharmacol. (1997) 12:137-40. doi: 10.1097/00004850-199705000-00003

36. Goldstein DJ, Lu Y, Detke MJ, Wiltse C, Mallinckrodt C, Demitrack MA. Duloxetine in the treatment of depression: a double-blind placebo-controlled comparison with paroxetine. J Clin Psychopharmacol. (2004) 24:389-99. doi: 10.1097/01.jcp.0000132448.65972.d9

37. Detke MJ, Lu Y, Goldstein DJ, Hayes JR, Demitrack MA. Duloxetine, $60 \mathrm{mg}$ once daily, for major depressive disorder: a randomized doubleblind placebo-controlled trial. J Clin Psychiatry. (2002) 63:308-15. doi: 10.4088/JCP.v63n0407

38. Goldstein DJ, Mallinckrodt C, Lu Y, Demitrack MA. Duloxetine in the treatment of major depressive disorder: a double-blind clinical trial. J Clin Psychiatry. (2002) 63:225-31. doi: 10.4088/JCP.v63n0309

39. Burt VK, Wohlreich MM, Mallinckrodt CH, Detke MJ, Watkin JG, Stewart DE. Duloxetine for the treatment of major depressive disorder in women ages 40 to 55 years. Psychosomatics. (2005) 46:345-54. doi: 10.1176/appi.psy.46.4.345

40. Perahia DG, Wang F, Mallinckrodt CH, Walker DJ, Detke MJ. Duloxetine in the treatment of major depressive disorder: a placeboand paroxetine-controlled trial. Eur Psychiatry. (2006) 21:367-78. doi: 10.1016/j.eurpsy.2006.03.004

41. Perahia DG, Gilaberte I, Wang F, Wiltse CG, Huckins SA, Clemens JW, et al. Duloxetine in the prevention of relapse of major depressive disorder: double-blind placebo-controlled study. Br J Psychiatry. (2006) 188:346-53. doi: 10.1192/bjp.188.4.346

42. Perahia DGS, Pritchett YL, Kajdasz DK, Bauer M, Jain R, Russell JM, et al. A randomized, double-blind comparison of duloxetine and venlafaxine in the treatment of patients with major depressive disorder. J Psychiatr Res. (2008) 42:22-34. doi: 10.1016/j.jpsychires.2007.01.008

43. Perahia DGS, Quail D, Desaiah D, Corruble E, Fava M. Switching to duloxetine from selective serotonin reuptake inhibitor antidepressants: a multicenter trial comparing 2 switching techniques. J Clin Psychiatry. (2008) 69:95-105. doi: 10.4088/JCP.v69n0113

44. Perahia DGS, Maina G, Thase ME, Spann ME, Wang F, Walker DJ, et al. Duloxetine in the prevention of depressive recurrences: a randomized, double-blind, placebo-controlled trial. J Clin Psychiatry. (2009) 70:706-16. doi: 10.4088/JCP.08m04756

45. Fava M, Detke MJ, Balestrieri M, Wang F, Raskin J, Perahia D. Management of depression relapse: re-initiation of duloxetine treatment or dose increase. J Psychiatr Res. (2006) 40:328-36. doi: 10.1016/j.jpsychires.2005.06.005

46. Brecht S, Courtecuisse C, Debieuvre C, Croenlein J, Desaiah D, Raskin J, et al. Efficacy and safety of duloxetine $60 \mathrm{mg}$ once daily in the treatment of pain in patients with major depressive disorder and at least moderate pain of unknown etiology: a randomized controlled trial. J Clin Psychiatry. (2007) 68:1707-16. doi: 10.4088/JCP.v68n1110

47. Lee P, Shu L, Xu X, Wang CY, Lee MS, Liu CY, et al. Once-daily duloxetine $60 \mathrm{mg}$ in the treatment of major depressive disorder: multicenter, doubleblind, randomized, paroxetine-controlled, non-inferiority trial in China, Korea, Taiwan and Brazil. Psychiatry Clin Neurosci. (2007) 61:295-307. doi: 10.1111/j.1440-1819.2007.01666.x

48. Brecht S, Desaiah D, Marechal ES, Santini AM, Podhorna J, Guelfi JD. Efficacy and safety of duloxetine $60 \mathrm{mg}$ and $120 \mathrm{mg}$ daily in patients hospitalized for severe depression: a double-blind randomized trial. J Clin Psychiatry. (2011) 72:1086-94. doi: 10.4088/JCP.09m05723blu

49. Pigott TA, Prakash A, Arnold LM, Aaronson ST, Mallinckrodt CH, Wohlreich MM. Duloxetine versus escitalopram and placebo: an 8-month, double-blind trial in patients with major depressive disorder. Curr Med Res Opin. (2007) 23:1303-18. doi: 10.1185/030079907X188107

50. Raskin J, Wiltse CG, Siegal A, Sheikh J, Xu J, Dinkel JJ, et al. Efficacy of duloxetine on cognition, depression, and pain in elderly patients with major depressive disorder: an 8-week, double-blind, placebo-controlled trial. Am J Psychiatry. (2007) 164:900-9. doi: 10.1176/ajp.2007.164.6.900

51. Raskin J, Wiltse CG, Dinkel JJ, Walker DJ, Desaiah D, Katona C. Safety and tolerability of duloxetine at $60 \mathrm{mg}$ once daily in elderly patients with major depressive disorder. J Clin Psychopharmacol. (2008) 28:32-8. doi: $10.1097 /$ jcp.0b013e318160738e

52. Wise TN, Wiltse CG, Iosifescu DV, Sheridan M, Xu JY, Raskin J. The safety and tolerability of duloxetine in depressed elderly patients with and without medical comorbidity. Int J Clin Pract. (2007) 61:1283-93. doi: $10.1111 / j .1742-1241.2007 .01476 . x$

53. Karp JF, Whyte EM, Lenze EJ, Dew MA, Begley A, Miller MD, et al. Rescue pharmacotherapy with duloxetine for selective serotonin reuptake inhibitor nonresponders in late-life depression: outcome and tolerability. $J$ Clin Psychiatry. (2008) 69:457-63. doi: 10.4088/JCP.v69n0317

54. Kornstein SG, Dunner DL, Meyers AL, Whitmyer VG, Mallinckrodt CH, Wohlreich MM, et al. A randomized, double-blind study of increasing or maintaining duloxetine dose in patients without remission of major depressive disorder after initial duloxetine therapy. J Clin Psychiatry. (2008) 69:1383-92. doi: 10.4088/JCP.v69n0905

55. Volpe FM. An 8-week, open-label trial of duloxetine for comorbid major depressive disorder and chronic headache. J Clin Psychiatry. (2008) 69:144954. doi: 10.4088/JCP.v69n0912

56. Herrera-Guzmán I, Herrera-Abarca JE, Gudayol-Ferré E, Herrera-Guzmán D, Gómez-Carbajal L, Peña-Olvira M, et al. Effects of selective serotonin reuptake and dual serotonergic-noradrenergic reuptake treatments on attention and executive functions in patients with major depressive disorder. Psychiatry Res. (2010) 177:323-29. doi: 10.1016/j.psychres.2010.03.006

57. Volonteri LS, Colasanti A, Cerveri G, Fiorentini A, De Gaspari IF, Mauri MC, et al. Clinical outcome and tolerability of duloxetine in the treatment of major depressive disorder: a 12-week study with plasma levels. J Psychopharmacol. (2010) 24:1193-9. doi: 10.1177/02698811091 04863

58. Sagman D, McIntosh D, Lee MS, Li H, Ruschel S, Hussain N, et al. Attributes of response in depressed patients switched to treatment with duloxetine. Int J Clin Pract. (2011) 65:73-81. doi: 10.1111/j.1742-1241.2010.02546.x

59. Gaynor PJ, Gopal M, Zheng W, Martinez JM, Robinson MJ, Marangell LB. A randomized placebo-controlled trial of duloxetine in patients with major depressive disorder and associated painful physical symptoms. Curr Med Res Opin. (2011) 27:1849-58. doi: 10.1185/03007995.2011.609539 
60. Martinez JM, Katon W, Greist JH, Kroenke K, Thase ME, Meyers AL, et al. A pragmatic 12-week, randomized trial of duloxetine versus generic selective serotonin-reuptake inhibitors in the treatment of adult outpatients in a moderate-to-severe depressive episode. Int Clin Psychopharmacol. (2012) 27:17-26. doi: 10.1097/YIC.0b013e32834ce11b

61. Oakes TMM, Myers AL, Marangell LB, Ahl J, Prakash A, Thase ME, et al. Assessment of depressive symptoms and functional outcomes in patients with major depressive disorder treated with duloxetine versus placebo: primary outcomes from two trials conducted under the same protocol. Hum Psychopharmacol. (2012) 27:47-56. doi: 10.1002/hup.1262

62. Rosso G, Rigardetto S, Bogetto F, Maina G. A randomized, singleblind, comparison of duloxetine with bupropion in the treatment of SSRI-resistant major depression. J Affect Disord. (2012) 136:172-6. doi: 10.1016/j.jad.2011.07.026

63. Robinson M, Oakes TM, Raskin JL, Liu P, Shoemaker S, Craig Nelson J. Acute and long-term treatment of late-life major depressive disorder: duloxetine versus placebo. Am J Geriatr Psychiatry. (2014) 22:34-45. doi: 10.1016/j.jagp.2013.01.019

64. Buoli M, Melter CC, Caldiroli A, Altamura AC. Are antidepressants equally effective in the long-term treatment of major depressive disorder? Hum Psychopharmacol. (2015) 30:21-7. doi: 10.1002/hup.2447

65. Mowla A, Dastgheib SA, Razeghian Jahromi L. Comparing the effects of sertraline with duloxetine for depression severity and symptoms: a doubleblind, randomized controlled trial. Clin Drug Investig. (2016) 36:539-43. doi: 10.1007/s40261-016-0399-6

66. De Donatis D, Florio V, Porcelli S, Saria A, Mercolini L, Serretti A, et al. Duloxetine plasma level and antidepressant response. Prog Neuro-Psychopharmacol Biol Psychiatry. (2019) 92:127-32. doi: 10.1016/j.pnpbp.2019.01.001

67. Alaka KJ, Noble W, Montejo A, Dueñas H, Munshi A, Strawn JR, et al. Efficacy and safety of duloxetine in the treatment of older adult patients with generalized anxiety disorder: a randomized, doubleblind, placebo-controlled trial. Int J Geriatr Psychiatry. (2014) 29:978-86. doi: $10.1002 /$ gps.4088

68. Bodkin JA, Allgulander C, Llorca PM, Spann ME, Walker DJ, Russell JM, et al. Predictors of relapse in a study of duloxetine treatment for patients with generalized anxiety disorder. Hum Psychopharmacol Clin Ext. (2011) 26:258-66. doi: 10.1002/hup.1211

69. Pierò A, Locati E. An open, non-randomised comparison of escitalopram and duloxetine for the treatment of subjects with generalized anxiety disorder. Hum Psychopharmacol Clin Ext. (2011) 26:63-71. doi: 10.1002/hup.1172

70. Wu WY, Wang G, Ball SG, Desaiah D, Ang QQ. Duloxetine versus placebo in the treatment of patients with generalized anxiety disorder in China. Chin Med J. (2011) 124:3260-8.

71. Nicolini H, Bakish D, Duenas H, Spann M, Erickson J, Hallberg C, et al. Improvement of psychic and somatic symptoms in adult patients with generalized anxiety disorder : examination from a duloxetine, venlafaxine extended-release and placebo-controlled trial. Psychol Med. (2009) 39:26776. doi: 10.1017/S0033291708003401

72. Allgulander C, Nutt D, Detke M, Erickson J, Spann M, Walker D, et al. A non-inferiority comparison of duloxetine and venlafaxine in the treatment of adult patients with generalized anxiety disorder. J Psychopharmacol. (2008) 22:417-25. doi: 10.1177/0269881108091588

73. Davidson J, Allgulander C, Pollack MH, Hartford J, Erickson JS, Russell $\mathrm{JM}$, et al. Efficacy and tolerability of duloxetine in elderly patients with generalized anxiety disorder: a pooled analysis of four randomized, doubleblind, placebo-controlled studies. Hum Psychopharmacol. (2008) 23:519-26. doi: 10.1002/hup.949

74. Pollack MH, Kornstein SG, Spann ME, Crits-Christoph P, Raskin J, Russell JM. Early improvement during duloxetine treatment of generalized anxiety disorder predicts response and remission at endpoint. J Psychiatr Res. (2008) 42:1176-84. doi: 10.1016/j.jpsychires.2008.02.002

75. Russell JM, Weisberg R, Fava M, Hartford JT, Erickson JS, D’Souza DN. Efficacy of duloxetine in the treatment of generalized anxiety disorder in patients with clinically significant pain symptoms. Depress Anxiety. (2008) 25:1-11. doi: 10.1002/da.20337
76. Rynn M, Russell J, Erickson J, Detke MJ, Ball S, Dinkel J, et al. Efficacy and safety of duloxetine in the treatment of generalized anxiety disorder: a flexible-dose, progressive-titration, placebo-controlled trial. Depress Anxiety. (2008) 25:182-9. doi: 10.1002/da.20271

77. Hartford J, Kornstein S, Liebowitz M, Pigott T, Russell J, Detke M, et al. Duloxetine as an SNRI treatment for generalized anxiety disorder : results from a placebo and active-controlled trial. Int Clin Psychopharmacol. (2007) 22:167-74. doi: 10.1097/YIC.0b013e32807fb1b2

78. Salehifar E, Janbabaei G, Hendouei N, Alipour A, Tabrizi N, Avan R. Comparison of the efficacy and safety of pregabalin and duloxetine in taxane-induced sensory neuropathy: a randomized controlled trial. Clin Drug Investig. (2020) 40:249-57. doi: 10.1007/s40261-019-00882-6

79. Jha S, Sahani OP, Siddiqui S, Verma MK, Mazumder A, Waghdhare S. Effectiveness of pregabalin compared to duloxetine in diabetic peripheral neuropathic pain: an observational study. J Assoc Physicians India. (2019) 67:32-6.

80. Farshchian N, Alavi A, Heydarheydari S, Moradian N. Comparative study of the effects of venlafaxine and duloxetine on chemotherapy-induced peripheral neuropathy. Cancer Chemother Pharmacol. (2018) 82:787-93. doi: 10.1007/s00280-018-3664-y

81. Schukro RP, Oehmke MJO, Geroldinger A, Heinze G, Kress H, Pramhas S. Efficacy of duloxetine in chronic low back pain with a neuropathic component a randomized, double-blind, placebo-controlled crossover trial. Anesthesiology. (2016) 124:150-8. doi: 10.1097/ALN.0000000000000902

82. Yasuda H, Hotta N, Kasuga M, Kashiwagi A, Kawamori R, Yamada T, et al. Efficacy and safety of $40 \mathrm{mg}$ or $60 \mathrm{mg}$ duloxetine in Japanese adults with diabetic neuropathic pain: results from a randomized, 52-week, open-label study. J Diabetes Investig. (2016) 7:100-8. doi: 10.1111/jdi.12361

83. Gao Y, Guo X, Han P, Li Q, Yang G, Qu S, et al. Treatment of patients with diabetic peripheral neuropathic pain in China: a double-blind randomised trial of duloxetine vs. placebo. Int J Clin Pract. (2015) 69:957-66. doi: 10.1111 ijcp.12641

84. Happich M, Schneider E, Boess FG, Wilhelm S, Schacht A, Birklein F, et al. Effectiveness of duloxetine compared with pregabalin and gabapentin in diabetic peripheral neuropathic pain results from a german observational study. Clin J Pain. (2014) 30:875-85. doi: 10.1097/AJP.0000000000000057

85. Irving G, Tanenberg RJ, Raskin J, Risser RC, Malcolm S. Comparative safety and tolerability of duloxetine vs. pregabalin vs duloxetine plus gabapentin in patients with diabetic peripheral neuropathic pain. Int J Clin Pract. (2014) 68:1130-40. doi: 10.1111/ijcp.12452

86. Vollmer TL, Robinson MJ, Risser RC, Malcolm SK. A randomized, double-blind, placebo-controlled trial of duloxetine for the treatment of pain in patients with multiple sclerosis. Pain Pract. (2014) 14:732-44. doi: $10.1111 /$ papr. 12127

87. Smith EML, Pang H, Cirrincione C, Felishman S, Paskett ED, Bressler LR, et al. Effect of duloxetine on pain, function, and quality of life among patients with chemotherapy-induced painful peripheral neuropathy: a randomized clinical trial. JAMA. (2013) 309:1359-67. doi: 10.1001/jama.2013.2813

88. Tesfaye S, Wilhelm S, Lledo A, Schacht A, Tölle T, Bouhassira D, et al. Duloxetine and pregabalin: high-dose monotherapy or their combination? the "cOMBO-DN study" - a multinational, randomized, double-blind, parallel-group study in patients with diabetic peripheral neuropathic pain. Pain. (2013) 154:2616-25. doi: 10.1016/j.pain.2013.05.043

89. Boyle J, Eriksson MEV, Gribble L, Gouni R, Johnsen S, Coppini DV, et al. Randomized, placebo-controlled comparison of amitriptyline, duloxetine, and pregabalin in patients with chronic diabetic peripheral neuropathic pain: impact on pain, polysomnographic sleep, daytime functioning, and quality of life. Diabetes Care. (2012) 35:2451-8. doi: 10.2337/dc12-0656

90. Tanenberg RJ, Irving GA, Risser RC, Ahl J, Robinson MJ, Skljarevski V, et al. Duloxetine, pregabalin, and duloxetine plus gabapentin for diabetic peripheral neuropathic pain management in patients with inadequate pain response to gabapentin: an open-label, randomized, noninferiority comparison. Mayo Clin Proc. (2011) 86:615-26. doi: 10.4065/mcp.2010.0681

91. Skljarevski V, Desaiah D, Chappell AS, Detke MJ, Gross JL, Ziegler D. Evaluating the maintenance of effect of duloxetine in patients with diabetic peripheral neuropathic pain. Diabetes Metab Res Rev. (2009) 25:623-31. doi: 10.1002/dmrr.1000 
92. Armstrong DG, Chappell AS, Le TK, Kajdasz DK, Backonja M, D'Souza $\mathrm{DN}$, et al. Duloxetine for the management of diabetic peripheral neuropathic pain: Evaluation of functional outcomes. Pain Med. (2007) 8:410-8. doi: 10.1111/j.1526-4637.2007.00276.x

93. Wernicke JF, Wang F, Pritchett YL, Smith TR, Raskin J, Souza DND, et al. An open-label 52-week clinical extension comparing duloxetine with routine care in patients with diabetic. Pain Med. (2007) 8:503-13. doi: 10.1111/j.1526-4637.2006.00258.x

94. Raskin J, Wang F, Pritchett YL, Goldstein DJ. Duloxetine for patients with diabetic peripheral neuropathic pain: a 6-month open-label safety study. Pain Med. (2006) 7:373-85. doi: 10.1111/j.1526-4637.2006.00207.x

95. Goldstein DJ, Lu Y, Detke MJ, Lee TC, Iyengar S. Duloxetine vs. placebo in patients with painful diabetic neuropathy. Pain. (2005) 116:109-18. doi: 10.1016/j.pain.2005.03.029

96. Raskin J, Pritchett YL, Wang F, D'Souza DN, Waninger AL, Iyengar S, et al. A double-blind, randomized multicenter trial comparing duloxetine with placebo in the management of diabetic peripheral neuropathic pain. Pain Med. (2005) 6:346-56. doi: 10.1111/j.1526-4637.2005.00061.x

97. Murakami M, Osada K, Ichibayashi H, Mizuno H, Ochiai T, Ishida M, et al. An open-label, long-term, phase III extension trial of duloxetine in Japanese patients with fibromyalgia. Mod Rheumatol. (2017) 27:688-95. doi: 10.1080/14397595.2016.1245237

98. Murakami M, Osada K, Mizuno H, Ochiai T, Alev L, Nishioka K. A randomized, double-blind, placebo-controlled phase III trial of duloxetine in Japanese fibromyalgia patients. Arthritis Res Ther. (2015) 17:1-13. doi: 10.1186/s13075-015-0718-y

99. Mohs R, Mease P, Arnold LM, Wang F, Ahl J, Gaynor PJ, et al. The effect of duloxetine treatment on cognition in patients with fibromyalgia. Psychosom Med. (2012) 74:628-34. doi: 10.1097/PSY.0b013e31825b9855

100. Arnold LM, Hudson JI, Wang F, Wohlreich MM, Prakash A, Kajdasz DK, et al. Comparisons of the efficacy and safety of duloxetine for the treatment of fibromyalgia in patients with versus without major depressive disorder. Clin J Pain. (2009) 25:461-8. doi: 10.1097/AJP.0b013e318197d4e4

101. Chappell AS, Littlejohn G, Kajdasz DK, Scheinberg M, D’souza DN, Moldofsky H. A 1-year safety and efficacy study of duloxetine in patients with fibromyalgia. Clin. J. Pain. (2009) 25:365-75. doi: 10.1097/AJP.0b013e31819be587

102. Russell IJ, Mease PJ, Smith TR, Kajdasz DK, Wohlreich MM, Detke MJ, et al. Efficacy and safety of duloxetine for treatment of fibromyalgia in patients with or without major depressive disorder: results from a 6-month, randomized, double-blind, placebo-controlled, fixed-dose trial. Pain. (2008) 136:432-44. doi: 10.1016/j.pain.2008.02.024

103. Arnold LM, Rosen A, Pritchett YL, D’Souza DN, Goldstein DJ, Iyengar S, et al. A randomized, double-blind, placebo-controlled trial of duloxetine in the treatment of women with fibromyalgia with or without major depressive disorder. Pain. (2005) 119:5-15. doi: 10.1016/j.pain.2005.06.031

104. Arnold LM, Lu Y, Crofford LJ, Wohlreich M, Detke MJ, Iyengar S, et al. A double-blind, multicenter trial comparing duloxetine with placebo in the treatment of fibromyalgia patients with or without major depressive disorder. Arthritis Rheum. (2004) 50:2974-84. doi: 10.1002/art.20485

105. Cornu JN, Merlet B, Ciofu C, Mouly S, Peyrat L, Sbe P, et al. Duloxetine for mild to moderate postprostatectomy incontinence: Preliminary results of a randomised, placebo-controlled trial. Eur Urol. (2011) 59:148-54. doi: 10.1016/j.eururo.2010.10.031

106. Cardozo L, Lange R, Voss S, Beardsworth A, Manning M, Viktrup L, et al. Short-and long-term efficacy and safety of duloxetine in women with predominant stress urinary incontinence. Curr Med Res Opin. (2010) 26:253-61. doi: 10.1185/03007990903438295

107. Bent AE, Gousse AE, Hendrix SL, Klutke CG, Monga AK, Yuen CK, et al. Duloxetine compared with placebo for the treatment of women with mixed urinary incontinence. Neurourol Urodyn. (2008) 27:212-21. doi: 10.1002/nau.20471

108. Lin ATL, Sun MJ, Tai HL, Chuang YC, Huang ST, Wang N, et al. Duloxetine versus placebo for the treatment of women with stress predominant urinary incontinence in Taiwan: a double-blind, randomized, placebo-controlled trial. BMC Urol. (2008) 8:1-9. doi: 10.1186/1471-2490-8-2

109. Schagen van Leeuwen JH, Lange RR, Jonasson AF, Chen WJ, Viktrup L. Efficacy and safety of duloxetine in elderly women with stress urinary incontinence or stress-predominant mixed urinary incontinence. Maturitas. (2008) 60:138-47. doi: 10.1016/j.maturitas.2008.04.012

110. Castro-Diaz D, Palma PCR, Bouchard C, Haab F, Hampel C, Carone R, et al. Effect of dose escalation on the tolerability and efficacy of duloxetine in the treatment of women with stress urinary incontinence. Int Urogynecol J. (2007) 18:919-29. doi: 10.1007/s00192-006-0256-x

111. Schlenker B, Gratzke C, Reich O, Schorsch I, Seitz M, Stief CG. Preliminary results on the off-label use of duloxetine for the treatment of stress incontinence after radical prostatectomy or cystectomy. Eur Urol. (2006) 49:1075-8. doi: 10.1016/j.eururo.2006.01.038

112. Weinstein DL, Cohen JS, Liu C, Meadows ES, Plouffe L, Muram D. Duloxetine in the treatment of women with stress urinary incontinence: results from DESIRE (Duloxetine Efficacy and Safety for Incontinence in Racial and Ethnic populations). Curr Med Res Opin. (2006) 22:2121-9. doi: 10.1185/030079906X148337

113. Ghoniem GM, Van Leeuwen JS, Elser DM, Freeman RM, Zhao YD, Yalcin I, et al. A randomized controlled trial of duloxetine alone, pelvic floor muscle training alone, combined treatment and no active treatment in women with stress urinary incontinence. J Urol. (2005) 173:1647-53. doi: 10.1097/01.ju.0000154167.90600.c6

114. Kinchen KS, Obenchain R, Swindle R. Impact of duloxetine on quality of life for women with symptoms of urinary incontinence. Int Urogynecol J. (2005) 16:337-44. doi: 10.1007/s00192-004-1270-5

115. Cardozo L, Drutz HP, Baygani SK, Bump RC. Pharmacological treatment of women awaiting surgery for stress urinary incontinence. Obstet Gynecol. (2004) 104:511-9. doi: 10.1097/01.AOG.0000134525.86480.0f

116. Millard RJ, Moore K, Rencken R, Yalcin I, Bump RC. Duloxetine vs placebo in the treatment of stress urinary incontinence: a fourcontinent randomized clinical trial. BJU Int. (2004) 93:311-8. doi: 10.1111/j.1464-410X.2004.04607.x

117. Van Kerrebroeck P, Abrams P, Lange R, Slack M, Wyndaele JJ, Yalcin I, et al. Duloxetine versus placebo in the treatment of European and Canadian women with stress urinary incontinence. BJOG An Int J Obstet Gynaecol. (2004) 111:249-57. doi: 10.1111/j.1471-0528.2004.00067.x

118. Dmochowski RR, Miklos JR, Norton PA, Zinner NR, Yalcin I, Bump RC. Duloxetine versus placebo for the treatment of North American women with stress urinary incontinence. J Urol. (2003) 170:1259-63. doi: 10.1097/01.ju.0000080708.87092.cc

119. Bradley LA, Wohlreich MM, Wang F, Gaynor PJ, Robinson MJ, D'Souza DN, et al. Pain response profile of patients with fibromyalgia treated with duloxetine. Clin J Pain. (2010) 26:498-504. doi: 10.1097/AJP.0b013e3181dee80e

120. Moher D, Liberati A, Tetzlaff J, Altman D. Preferred reporting items for systematic reviews and metaanalyses: the PRISMA Statement. Ann Intern Med. (2009) 151:264-9. doi: 10.7326/0003-4819-151-4-20090818000135

121. Munn Z, Moola S, Riitano D, Lisy K. The development of a critical appraisal tool for use in systematic reviews addressing questions of prevalence. Int J Heal Policy Manag. (2014) 3:123-8. doi: 10.15171/ijhpm.2014.71

122. Bigby M, Williams H. Appraising systematic reviews and metaanalyses. Arch Dermatol. (2003) 139:795-8. doi: 10.1001/archderm.139. 6.795

123. Naranjo C, Del Reguero L, Moratalla G, Hercberg M, Valenzuela M, Failde I. Anxiety, depression and sleep disorders in patients with diabetic neuropathic pain: a systematic review. Expert Rev Neurother. (2019) 19:1201-9. doi: 10.1080/14737175.2019.1653760

124. Vileikyte L, Leventhal H, Gonzalez JS, Peyrot M, Rubin RR, Ulbrecht JS, et al. Diabetic peripheral neuropathy and depressive symptoms: the association revisited. Diabetes Care. (2005) 28:2378-83. doi: 10.2337/diacare.28. 10.2378

125. Arnold LM, Hudson JI, Keck PE, Auchenbach MB, Javaras KN, Hess EV Comorbidity of fibromyalgia and psychiatric disorders. J Clin Psychiatry. (2006) 67:1219-25. doi: 10.4088/JCP.v67n0807

126. Heymen S. Psychological and cognitive variables affecting treatment outcomes for urinary and fecal incontinence. Gastroenterology. (2004) 126:S146-51. doi: 10.1053/j.gastro.2003.10.040

127. Obata H. Analgesic mechanisms of antidepressants for neuropathic pain. Int J Mol Sci. (2017) 18:1-12. doi: 10.3390/ijms18112483 
128. Haase J, Brown E. Integrating the monoamine, neurotrophin and cytokine hypotheses of depression - a central role for the serotonin transporter? Pharmacol Ther. (2015) 147:1-11. doi: 10.1016/j.pharmthera.2014. 10.002

129. Montoya A, Bruins R, Katzman MA, Blier P. The noradrenergic paradox: implications in the management of depression and anxiety. Neuropsychiatr Dis Treat. (2016) 12:541-57. doi: 10.2147/NDT.S91311

130. Liu Y, Zhao J, Fan X, Guo W. Dysfunction in serotonergic and noradrenergic systems and somatic symptoms in psychiatric disorders. Front Psychiatry. (2019) 10:286. doi: 10.3389/fpsyt.2019.00286

131. Nekovarova T, Yamamotova A, Vales K, Stuchlik A, Fricova J, Rokyta R. Common mechanisms of pain and depression: are antidepressants also analgesics? Front Behav Neurosci. (2014) 8:99. doi: 10.3389/fnbeh.2014. 00099

132. Miyazato M, Kaiho Y, Kamo I, Chancellor MB, Sugaya K, De Groat WC, et al. Effect of duloxetine, a norepinephrine and serotonin reuptake inhibitor, on sneeze-induced urethral continence reflex in rats. Am J Physiol - Ren Physiol. (2008) 295:F264-71. doi: 10.1152/ajprenal.9024 1.2008

133. Sultan A, Gaskell H, Derry S, Andrew RA. Duloxetine for painful diabetic neuropathy and fibromyalgia pain: systematic review of randomised trials. BMC Neurol. (2008) 8:29. doi: 10.1186/1471-2377-8-29

134. Karpa KD, Cavanaugh JE, Lakoski JM. Duloxetine pharmacology: Profile of a dual monoamine modulator. CNS Drug Rev. (2002) 8:361-76. doi: 10.1111/j.1527-3458.2002.tb00234.x

135. Lisinski A, Hieronymus F, Näslund J, Nilsson S, Eriksson E. Itembased analysis of the effects of duloxetine in depression: a patientlevel post hoc study. Neuropsychopharmacology. (2019) 45:553-60. doi: 10.1038/s41386-019-0523-4

136. Sharma A, Goldberg MJ, Cerimele BJ. Pharmacokinetics and safety of duloxetine, a dual-serotonin and norepinephrine reuptake inhibitor. J Clin Pharmacol. (2000) 40:161-7. doi: 10.1177/009127000220 08810

137. Smith HS, Smith EJ, Smith BR. Duloxetine in the management of chronic musculoskeletal pain. Ther Clin Risk Manag. (2012) 8:267-77. doi: 10.2147/TCRM.S17428
138. De Heer EW, Gerrits MMJG, Beekman ATF, Dekker J, Van Marwijk HWJ, De Waal MWM, et al. The association of depression and anxiety with pain: a study from NESDA. PLoS ONE. (2014) 9:e106907. doi: 10.1371/journal.pone.0106907

139. Crocq MA. Alcohol, nicotine, caffeine, and mental disorders. Dialogues Clin Neurosci. (2003) 5:175-85.

140. Vinader-Caerols C, Monleón S, Carrasco C, Parra A. Effects of alcohol, coffee, and tobacco, alone or in combination, on physiological parameters and anxiety in a young population. J Caffeine Res. (2012) 2:70-6. doi: $10.1089 /$ jcr.2012.0018

141. Fric M, Pfuhlmann B, Laux G, Riederer P, Distler G, Artmann S, et al. The influence of smoking on the serum level of duloxetine. Pharmacopsychiatry. (2008) 41:151-5. doi: 10.1055/s-2008-1073173

142. Vuppalanchi R, Hayashi PH, Chalasani N, Fontana RJ, Bonkovsky H, Saxena R, et al. Duloxetine hepatotoxicity: a case-series from the druginduced liver injury network. Aliment Pharmacol Ther. (2010) 32:1174-83. doi: $10.1111 /$ j.1365-2036.2010.04449.x

Conflict of Interest: JO declares paid positions, honoraria or being part of advisory boards by Angelini, AstraZeneca, Bristol-Myers, Casen Ricordati, Esteve, GSK, Janssen, Lilly, Lundbeck, Novartis, Otsuka, Pfizer and Sanofi, and grants from Ministry of Health Spanish National Institute of Heath Carlos III, National Substance Abuse Plan, and Galician Innovation Programs (GAIN) in the last 10 years.

The remaining authors declare that the research was conducted in the absence of any commercial or financial relationships that could be construed as a potential conflict of interest.

Copyright (c) 2020 Rodrigues-Amorim, Olivares, Spuch and Rivera-Baltanás. This is an open-access article distributed under the terms of the Creative Commons Attribution License (CC BY). The use, distribution or reproduction in other forums is permitted, provided the original author(s) and the copyright owner(s) are credited and that the original publication in this journal is cited, in accordance with accepted academic practice. No use, distribution or reproduction is permitted which does not comply with these terms. 